\title{
Connaissance des émissions gazeuses dans les différentes filières de gestion des effluents porcins
}

\author{
M. BONNEAU ${ }^{1}$, J.-Y. DOURMAD ${ }^{1}$, J.-C. GERMON², M. HASSOUNA ${ }^{3}$, B. LEBRET' \\ L. LOYON 4 , J.-M. PAILLAT', Y. RAMONET', P. ROBIN ${ }^{3}$ \\ ${ }^{1}$ INRA, UMR 1079 Systèmes d'Elevage Nutrition Animale et Humaine, F-35590 Saint-Gilles, France \\ ${ }^{2}$ INRA, UMR 1229 Microbiologie du sol et de l'Environnement, F-21065 Dijon, France \\ ${ }^{3}$ INRA, UMR 1069 Sol, Agro-hydrosystèmes, Spatialisation, F-35042 Rennes, France \\ ${ }^{4}$ Cemagref, UR Gestion environnementale et traitement biologique des déchets, F-35044 Rennes, France \\ ${ }^{5}$ CIRAD, UPR Recyclage et Risque, F-35042 Rennes, France \\ ${ }^{6}$ Chambre Régionale d'Agriculture de Bretagne, F-35042 Rennes, France \\ Courriel : Michel.Bonneau@rennes.inra.fr
}

Le programme «Porcherie verte» a consenti un effort particulier pour mieux connaître les émissions gazeuses associées à l'élevage porcin et qui peuvent avoir un impact sur l'environnement ou la santé de l'Homme (cf. tableau 1 de l'introduction générale). Jusqu'alors les mesures s'étaient essentiellement focalisées sur 1'ammoniac, dont les effets délétères sur la santé des animaux sont bien établis, dans les filières «Lisier». Nous nous sommes attachés à élargir la palette des mesures en y intégrant les gaz à effet de serre et à quantifier les émissions gazeuses, très mal connues jusqu'alors, dans les filières gérant des effluents solides (litières, composts).

La plupart des publications issues du programme «Porcherie verte» présentent les résultats de façon horizontale, en se focalisant sur une étape particulière des filières de gestion des effluents. Dans cet article, nous avons pris le parti de présenter les résultats par élément gazeux (ammoniac $\mathrm{NH}_{3}$, protoxyde d'azote $\mathrm{N}_{2} \mathrm{O}$ aussi appelé oxyde nitreux, dioxyde de carbone $\mathrm{CO}_{2}$ et méthane $\mathrm{CH}_{4}$ ), de façon à rapprocher les résultats obtenus aux différents niveaux des filières de gestion des effluents. La dernière section de cet article essaie de faire la synthèse des connaissances acquises sur les émissions de gaz azotés. Elle s'appuie pour ce faire sur les figures 9 à 12 qui tentent de montrer comment le programme «Porcherie verte» a contribué à faire avancer les connaissances dans le domaine. Nous renvoyons le lecteur aux autres articles de ce numéro pour la présentation des résultats relatifs aux éléments à risques (azote, phosphore et éléments traces métalliques) ou aux nuisances olfactives ou pour des approches plus globalisantes.

Nous avons voulu caractériser certains facteurs de variation des émissions d'ammoniac $\left(\mathrm{NH}_{3}\right)$ dans les bâtiments utilisant la filière "Lisier». Pour diminuer ces émissions on peut jouer sur différents tableaux : réduire la teneur en protéines des aliments distribués aux animaux (cf. §1.2), éviter le stockage du lisier sous les animaux par un renouvellement pluriquotidien (cf. §1.4), réduire la fréquence des brassages du lisier stocké dans les fosses (cf. §1.5). Le traitement biologique des lisiers conduit aussi à une réduction globale des émissions de $\mathrm{NH}_{3}$, surtout en l'absence de séparation de phases (cf. §1.7). Les émissions de $\mathrm{NH}_{3}$ par les litières sont extrêmement variables en fonction de la nature du substrat utilisé et de son épaisseur (cf. §1.3). De même les émissions associées au compostage peuvent varier fortement en fonction de la nature des effluents compostés et de la technique employée (cf. §1.6).

Les processus de nitrification/dénitrification qui ont cours dans les litières, lors du traitement biologique et du compostage, ainsi que dans les sols, conduisent à volatiliser une partie de l'azote présent, majoritairement sous forme de diazote $\left(\mathrm{N}_{2}\right)$, mais aussi sous forme de protoxyde d'azote $\left(\mathrm{N}_{2} \mathrm{O}\right)$, dans une proportion très variable et asse $z$ mal connue. Or le $\mathrm{N}_{2} \mathrm{O}$ est un gaz à effet de serre très puissant. Nous avons montré que les quantités de $\mathrm{N}_{2} \mathrm{O}$ émises lors du traitement biologique des lisiers dépend fortement des techniques d'aération mises en œuvre (cf. §2.3). Elles sont de façon générale plus élevées lorsque les animaux sont élevés sur litières que sur caillebotis, mais des variations très importantes sont observées en fonction du mode de conduite des litières (cf. §2.1). Nous avons montré aussi que le compostage en couche mince, préconisé pour économiser la paille, entraîne des émissions accrues de $\mathrm{N}_{2} \mathrm{O}$ (cf. \$2.2). Enfin les émissions de $\mathrm{N}_{2} \mathrm{O}$ dans le sol après épandage ne semblent pas différer entre azote organique apporté par des effluents d'élevage et azote apporté par les engrais minéraux (cf. \$2.4). Dans les deux cas, ces émissions sont très difficiles à caractériser car extrêmement sensibles aux conditions pédoclimatiques.

Le carbone de la matière organique des effluents et des éventuels substrats ajoutés est également susceptible d'être en partie volatilisé, plutôt sous forme de dioxyde de carbone $\left(\mathrm{CO}_{2}\right)$ en conditions aérobies, plutôt sous forme de méthane $\left(\mathrm{CH}_{4}\right)$ en conditions anaérobies. Les deux gaz contribuent à l'effet de serre mais le second bien davantage que le premier. Nous avons établi que le logement des animaux sur litière conduisait à des émissions de $\mathrm{CH}_{4}$ plus faibles que sur caillebotis (cf. \$4.1). Nous avons montré que le compostage en couche mince conduit à des émissions de $\mathrm{CO}_{2}$ plus faibles (cf. \$3.1) mais de $\mathrm{CH}_{4}$ plus élevées (cf. §4.2) que les techniques de compostage classiques. Nous avons constaté enfin que le traitement biologique du lisier permettait de réduire les émissions de 
$\mathrm{CO}_{2}$, sous réserve qu'il n'y ait pas de séparation de phases (cf. \$3.2) et de $\mathrm{CH}_{4}$ (cf. §4.3), par rapport à un simple stockage.

\section{1 / Les émissions d'ammo- niac}

Les émissions d'ammoniac $\left(\mathrm{NH}_{3}\right)$ proviennent de la volatilisation de l'azote ammoniacal $\left(\mathrm{NH}_{4}^{+}\right)$en solution dans les phases liquides des déjections. L'azote d'origine fécale, essentiellement sous forme de protéines, est relativement résistant à la décomposition, mais il ne représente qu'une fraction minoritaire de l'azote total des déjections. L'azote urinaire, qui représente la plus grande part de l'azote excrété par l'animal, est essentiellement sous forme d'urée, qui est rapidement dégradée en $\mathrm{NH}_{4}{ }^{+}$. Les déjections porcines constituent donc une source potentiellement importante de $\mathrm{NH}_{3}$, dont la production dépendra beaucoup des conditions dans lesquelles les déjections sont stockées et éventuellement traitées. Les émissions de $\mathrm{NH}_{3}$ dépendent ainsi de quatre grandes familles de facteurs, qui constituent autant de leviers d'action potentiels :

- la quantité d'azote urinaire, sensible à la composition des aliments distribués aux animaux ;

- l'ammonification de l'azote organique présent dans l'effluent ou dans la biomasse microbienne ;

- le processus de volatilisation qui est gouverné par le $\mathrm{pH}$, la température, le renouvellement d'air à la surface ainsi que par les surfaces et temps d'échange avec l'atmosphère ;

- la transformation de l'azote $\mathrm{NH}_{4}{ }^{+}$en d'autres composés azotés simples (nitrification) ou complexes (immobilisation).

\section{1 / Modélisation des émissions de $\mathrm{NH}_{3}$ dans les bâtiments d'éle- vage}

Dourmad et al (2002) ont développé un modèle qui permet de prédire les quantités de $\mathrm{NH}_{3}$ volatilisées dans le bâtiment d'élevage, en fonction des performances des animaux et des caractéristiques du bâtiment (tableau 1). Le coefficient de volatilisation (Coeff $\mathrm{Volat}_{\text {at }}$ ) appliqué aux quantités d'azote excrété par les animaux $\left(\mathrm{N}_{\text {excrété }}\right)$ est corrigé des influences de la concentration en azote ammoniacal (Effet ${ }_{\text {TNAm }}$ ) et de la température $\left(\mathrm{Effet}_{\mathrm{Temp}}\right) \mathrm{du}$ lisier, du taux de renouvellement de l'air $\left(\right.$ Effet $\left._{\text {Renouv }}\right)$, du type de sol (Effet ${ }_{\text {Sol }}$ ) et du rythme d'évacuation du lisier du bâtiment
Tableau 1. Equations de prédiction des quantités de $\mathrm{NH}_{3}$ volatilisées dans un bâtiment d'élevage porcin sur caillebotis (d'après Dourmad et al 2002).

\section{Excrétion azotée}

$$
\begin{aligned}
& \mathrm{N}_{\text {Excrété }}=\mathrm{N}_{\text {Ingéré }}-\mathrm{N}_{\text {Retenu }} \\
& \mathrm{N}_{\text {Retenu }}=\mathrm{N}_{\text {Corporel }}(\text { final })-\mathrm{N}_{\text {Corporel }}(\text { initial }) \\
& N_{\text {Corporel }}=e^{(-0,9892-0,0145(\text { TVM - 3)) }}\left(0,915 \text { Poids }_{\text {Vif }}{ }^{1,009}\right)^{(0,7518+0,0044(\text { TVM - 3)) }} / 6,25 \\
& \mathrm{~N}_{\text {Fèces }}=\text { Aliment } \times \mathrm{MS} \times(1-\mathrm{CudN}) \\
& \text { CudN }_{\text {Croissance }}=(-0,128+(7,80 \text { ED + 0,87 MAT) } / \text { MS }) /(\text { MAT / MS }) \\
& \text { CudN }_{\text {Truie }}=(-0,108+(6,17 \mathrm{ED}+0,92 \mathrm{~PB}) / \mathrm{MS}) /(\mathrm{MAT} / \mathrm{MS})
\end{aligned}
$$

$\mathrm{N}_{\text {Urinaire }}=\mathrm{N}_{\text {Excrété }}-\mathrm{N}_{\text {Fèces }}$

\section{Volatilisation d'N ammoniacal}

$N_{\text {Volatilisé }}=$ Coeff $_{\text {volat }} \times \mathrm{N}_{\text {Excrété }}$

Coeff $_{\text {Volat }}=0,24 \times$ Effet $_{\text {TNam }} \times$ Effet $_{\text {Temp }} \times$ Effet $_{\text {Rnouv }} \times$ Effet $_{\text {Ventil. }} \times$ Effetsol $_{\text {Soffet }} \times$ Eythme

Effet $_{\mathrm{TNam}}=1+0,21\left(\mathrm{TN}_{\mathrm{Am}}-0,51\right)$

$\mathrm{TN}_{\text {Am }}(\mathrm{Mol} / \mathrm{kg})=\mathrm{N}_{\text {Urinaire }} / 14$

Effet $_{\text {Temp }}=1+0,053\left(\right.$ Temp $\left._{\text {Lisier }}-22\right)$

Temp $_{\text {Lisier }}=-0,012$ Tempambiante $^{2}+1,1816$ Temp $_{\text {ambiante }}+1,6064$

Effet $_{\text {Renouv }}=1+0,636\left(\right.$ TauX $\left._{\text {Renouv }}-0,6\right)$

Effetsol $_{1,00}$ : caillebotis béton total $-0,85$ : caillebotis métallique total $-0,80$ : caillebotis partiel

Effet $_{\text {Rythme }}=1,00:>4$ semaines $-0,90: 2$ semaines $-0,80: 1$ semaine $-0,65$ : $<1$ jour

$\left(\right.$ Effet $\left._{\text {Rythme }}\right)$. Le modèle ne prend cependant pas en compte la volatilisation intervenant pendant les phases de stockage hors bâtiment, de traitement et d'épandage. Par ailleurs, il n'est applicable qu'aux bâtiments sur caillebotis, à l'exclusion de ceux utilisant de la litière.

Le modèle décrit dans cette présentation est une première étape d'un modèle plus global qui intégrera également les facteurs de variation liés au stockage et au traitement des effluents, ainsi que d'autres formes de collecte comme les litières. Des interfaces avec des modèles agronomiques sont également envisagées. La vocation de ce modèle est d'être un outil «ouvert» utilisable pour quantifier les risques environnementaux et améliorer la gestion des effluents au sein de l'exploitation.

\section{2 / La diminution de la teneur en protéines de l'aliment distri- bué aux animaux conduit à une réduction des émissions de $\mathrm{NH}_{3}$}

La teneur en protéines de l'aliment distribué aux animaux influence fortement les caractéristiques des lisiers (Portejoie 2002, Portejoie et al 2002, 2004). La réduction de $20 \%$ à $12 \%$ de la teneur en protéines des aliments dis- tribués aux animaux permet de diminuer sensiblement la quantité de lisier (de 6,4 à 4,7 kg par jour et par porc) et sa teneur en azote total et ammoniacal (de 4,3 à 1,9 g N par kg de lisier). Le $\mathrm{pH}$ du lisier est également abaissé de 8,9 à 7,6 . Tous ces éléments concourent à ce que les émissions de $\mathrm{NH}_{3}$ soient sensiblement réduites aussi bien pendant le stockage (figure 1) du lisier que

Figure 1. Cinétique de volatilisation $d u$ $\mathrm{NH}_{3}$ pendant le stockage de 3 types de lisiers différant par la teneur en protéines de l'aliment distribué aux animaux (quantités cumulées, d'après Portejoie et al 2004).

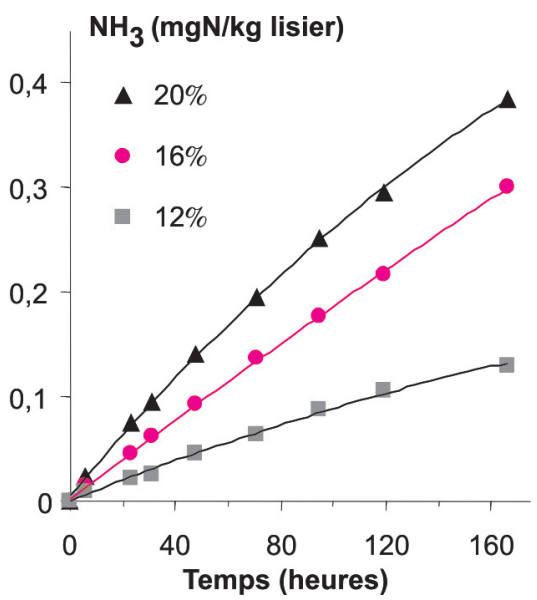


Figure 2. Cinétique de volatilisation à l'épandage de 3 types de lisiers différant par la teneur en protéines de l'aliment distribué aux animaux (quantités cumulées, d'après Portejoie et al 2004).

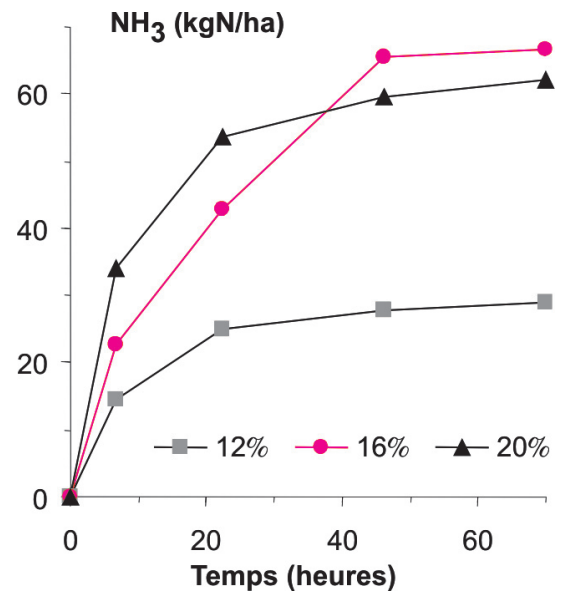

lors de son épandage (figure 2). Au total les quantités de $\mathrm{NH}_{3}$ volatilisées par porc sont réduites d'un facteur 3 lorsque la teneur en protéines de l'aliment est abaissée de $20 \%$ à $12 \%$ et d'un facteur 2 lorsqu'elles sont réduites de $16 \%$ à $12 \%$. Sous réserve de compenser la baisse de la teneur en protéines par un apport d'acides aminés purifiés, les trois régimes permettent les mêmes performances zootechniques.

\section{3 / Les émissions d'ammoniac varient en fonction du substrat utilisé pour la litière et de son mode de conduite}

Lors de la comparaison expérimentale de deux systèmes de logement fortement contrastés, Lebret et al (2004) observent des émissions de $\mathrm{NH}_{3}$ assez similaires pour un caillebotis intégral et une litière de sciure profonde avec accès à une courette extérieure bétonnée. Les concentrations de $\mathrm{NH}_{3}$ à l'intérieur des deux bâtiments sont également sensiblement les mêmes $\left(10,2 \mathrm{mg} / \mathrm{m}^{3}\right.$ pour la litière contre $11,0 \mathrm{mg} / \mathrm{m}^{3}$ pour le caillebotis). Ce résultat n'est cependant applicable qu'au cas particulier des deux situations comparées dans cet essai. Ainsi, Kermarrec et Robin (2002), dans une autre situation expérimentale, observent des émissions de $\mathrm{NH}_{3}$ deux fois plus élevées sur caillebotis que sur litière de sciure profonde. De nombreux facteurs peuvent en effet faire varier les taux d'émissions de $\mathrm{NH}_{3}$. Ces facteurs sont assez bien connus pour les élevages sur caillebotis (cf. §1.1, Dourmad et al 2002).
Dans le cas des élevages sur litière, les émissions de gaz peuvent varier fortement en fonction du climat, du substrat utilisé (nature, quantité et fréquence des apports), de la profondeur de la litière et de la densité animale. On sait que l'abattement d'azote (pourcentage de l'azote initialement présent dans les excréments animaux qui est volatilisé sous diverses formes gazeuses) est plus important avec un substrat de sciure $(72 \pm 7 \%)$ qu'avec de la paille $(57 \pm 13 \%$; CORPEN 2003$)$, mais l'équilibre entre les fractions volatilisées sous forme d'azote atmosphérique $\left(\mathrm{N}_{2}\right)$, de $\mathrm{NH}_{3}$ et de $\mathrm{N}_{2} \mathrm{O}$ peuvent être très différents selon les situations.

Les premières observations de Ramonet et Robin (2002) montraient que les litières de sciure en couche fine volatilisent $60 \%$ de l'azote excrété par les animaux, essentiellement sous forme de $\mathrm{NH}_{3}$. Ceci peut conduire à des concentrations élevées de $\mathrm{NH}_{3}$ dans les bâtiments, nuisibles à la santé des animaux et des travailleurs. Les observations ultérieures des mêmes auteurs (Ramonet et Robin 2005) confirment les forts taux d'abattement de l'azote sur litières de sciure ou de copeaux, plus élevés que pour les litières de paille. Dans le cas de la sciure et dans des conditions de conduite de litière mieux maîtrisées que lors des premières observations, ils font état d'émissions de $\mathrm{NH}_{3}$ plus faibles, représentant $19 \%$ de l'azote excrété par les animaux. Cette dernière observation souligne l'importance de bien gérer les litières en couche fine de sciure. Des apports de sciure fraîche sont à effectuer dès que la litière devient trop humide.

En litière de paille profonde, les émissions de $\mathrm{NH}_{3}$ représentent de 15 à $25 \%$ de l'azote excrété par les animaux avec un taux de paillage compris entre 50 et $80 \mathrm{~kg}$ paille/porc (Robin et al 2004) et une surface disponible de 1,0 à $1,4 \mathrm{~m}^{2}$ par porc. Toutes conditions égales par ailleurs, une réduction de la densité animale, au-delà de $2 \mathrm{~m}^{2}$ par porc, conduit à des émissions de $\mathrm{NH}_{3}$ plus faibles, comprises entre 5 et $15 \%$ de l'azote excrété par les animaux. Une augmentation du paillage conduit aussi à une diminution des émissions de $\mathrm{NH}_{3}$. Ces résultats couplés à ceux obtenus sur le compostage (cf. §2.1.f) montrent l'existence d'un optimum d'humidité pour que les fermentations microbiennes s'orientent favorablement. Si l'humidité est trop faible, la flore microbienne ne peut se développer et des pics d'ammoniac pourront être observés. Dans le cas inverse, la présence d'un excès d'eau réduit la porosité libre à l'air et les apports d'oxygène sont insuffisants pour assurer la réorganisation de l'azote en protéines bactériennes. Cette situation peut ainsi conduire également à des émissions élevées d'ammoniac.

\section{4 / Le renouvellement fré- quent du lisier diminue les teneurs en $\mathrm{NH}_{3}$ dans les bâti- ments}

Les expérimentations conduites par Ramonet et al (2007) montrent que le nettoyage pluriquotidien des déjections par un effet de «chasse d'eau», utilisant du lisier recyclé après épuration, permet de diminuer de $70 \%$ les concentrations en $\mathrm{NH}_{3}$ à l'intérieur du bâtiment d'élevage. On peut donc penser que les émissions de $\mathrm{NH}_{3}$ sont fortement abaissées, mais ceci demanderait à être démontré en effectuant un bilan global sur l'ensemble de la chaîne de recyclage du lisier.

\section{5 / Le brassage du lisier dans les fosses de stockage favorise les émissions de $\mathrm{NH}_{3}$}

Des expérimentations effectuées au laboratoire ont montré que le brassage régulier du lisier double les quantités de $\mathrm{NH}_{3}$ émises dans la fosse de stockage (Thirion et al 2005). L'augmentation de ces émissions est principalement due à une augmentation du $\mathrm{pH}$ nettement plus importante lors d'un brassage régulier que lors d'un stockage sans brassage. Cette augmentation du $\mathrm{pH}$ est probablement liée au relargage du $\mathrm{CO}_{2}$ dissous. Si le lisier n'est homogénéisé qu'une seule fois, en fin de période de stockage, les émissions n'augmentent que de 10 à $30 \%$ par rapport à une absence totale de brassage. Cependant, une importante augmentation de $\mathrm{pH}$ est également observée avec un brassage uniquement en fin de période de stockage, ce qui est susceptible d'accroître les émissions ultérieures de $\mathrm{NH}_{3}$ au moment de l'épandage. Ces résultats nécessitent cependant d'être confirmés en conditions réelles de stockage. D'une part, le temps de stockage en conditions réelles est beaucoup plus long que le temps considéré lors de ces expérimentations. D'autre part, les fosses de stockage sont régulièrement alimentées en lisiers provenant des bâtiments d'élevage. 
1.6 / Les émissions de $\mathrm{NH}_{3}$ au cours du compostage dépendent des caractéristiques initiales du substrat composté, mais aussi de la technique de compostage

Les émissions de $\mathrm{NH}_{3}$ au cours du compostage de déjections porcines peuvent varier considérablement, représentant de 15 à $50 \%$ de l'azote initialement présent (Paillat et al 2005a, figure 3). Elles peuvent même dépasser $60 \%$ dans le cas de litières mal maîtrisées (tableau 2). Les réactions conduisant à la production de $\mathrm{NH}_{3}$ s'installent beaucoup plus vite dans les composts à base de fumier que dans ceux préparés avec du lisier (figure 4). Dans le cas des substrats à base de lisier, elles s'installent d'autant plus vite que la proportion de matière sèche
Figure 4. Temps nécessaire pour obtenir l'émission maximale d'ammoniac lors du compostage de déjections porcines variées sur divers substrats ( $d$ 'après Paillat et al 2005a).

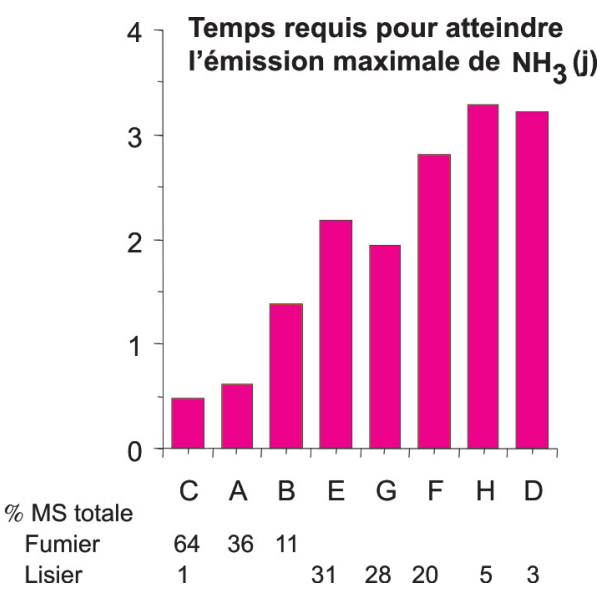

apportée par le lisier est plus élevée. Cette vitesse d'installation dépend en effet de la quantité initiale de flore bactérienne, que l'on peut considérer proportionnelle à la quantité de déjections animales présente et plus élevée dans un fumier que dans un lisier. Cependant, elle dépend également de la proportion de carbone biodégradable qui détermine la rapidité de montée en température. Dans tous les cas, l'essentiel du $\mathrm{NH}_{3}$ est émis au cours du premier mois de compostage, durant la phase thermophile (figure 3). Les quantités d'azote disponible et de carbone biodégradable, la porosité libre à l'air et l'humidité sont des facteurs déterminants de la dégradation des matières organiques et des émissions de gaz. L'émission de $\mathrm{NH}_{3}$ est plus élevée avec des substrats riches en azote disponible (figure 5) alors que la quantité de

Figure 3. Emissions cumulées de gaz après 28 ou 56 jours de compostage de déjections porcines variées sur divers substrats (B-C : fumiers ; $D-H$ : lisiers, $A$ : mélange fumier+lisier, d'après Paillat et al 2005a).
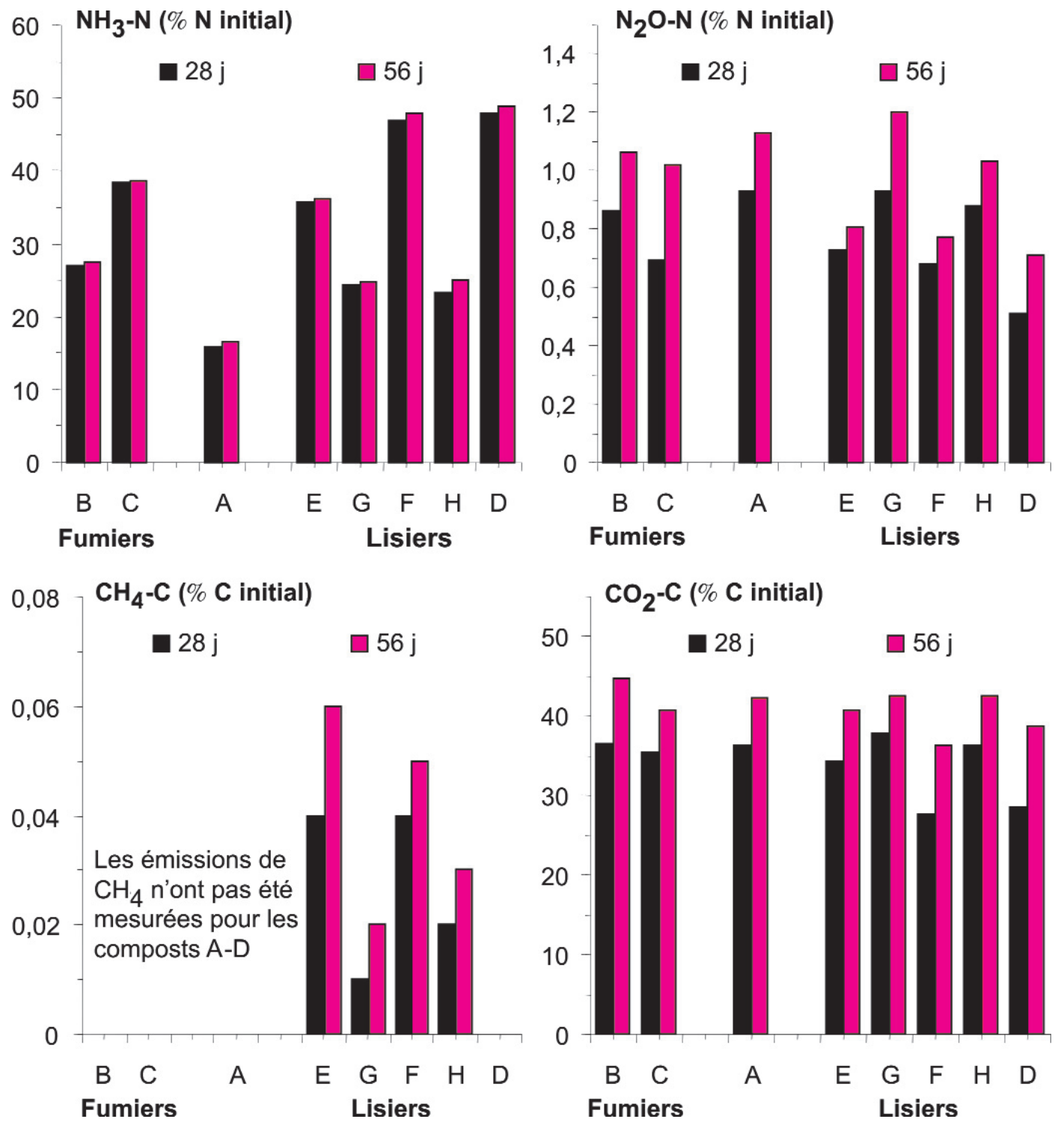
Figure 5. Influence des quantités d'azote disponible et de carbone biodégradable sur l'émission d'ammoniac lors du compostage (modélisation de l'émission cumulée à 56 jours, d'après Paillat et al 2005a).

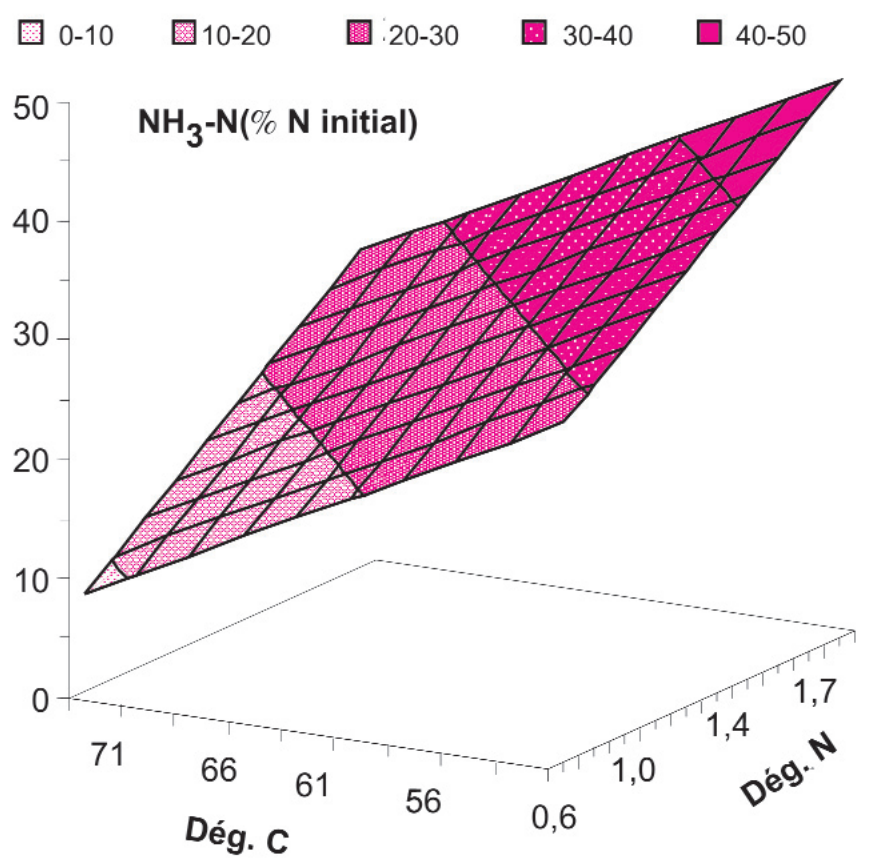

carbone biodégradable est au contraire un facteur défavorable. La durée de l'émission dépend de la proportion d'azote disponible. Ainsi, alors que la nature de l'effluent (fumier vs lisier) condi-

tionne la vitesse de dégagement du $\mathrm{NH}_{3}$, elle n'a pas d'effet direct sur les quantités totales émises, qui peuvent être prédites à partir de deux mesures simples, l'azote soluble à l'eau $\left(12 \mathrm{~h}\right.$ à $\left.4^{\circ} \mathrm{C}\right)$ qui rend

Figure 6. Emissions comparées de gaz en aval d'un élevage "naisseur-engraisseur» lors d'un simple stockage du lisier avant épandage (S), d'un traitement biologique sans séparation de phase $(T)$ ou de traitements biologiques avec séparation de phase à l'aide d'une centrifugeuse (TC) où d'une vis compacteuse (TV). La phase d'épandage n'est pas prise en considération (d'après Loyon et al 2006b).

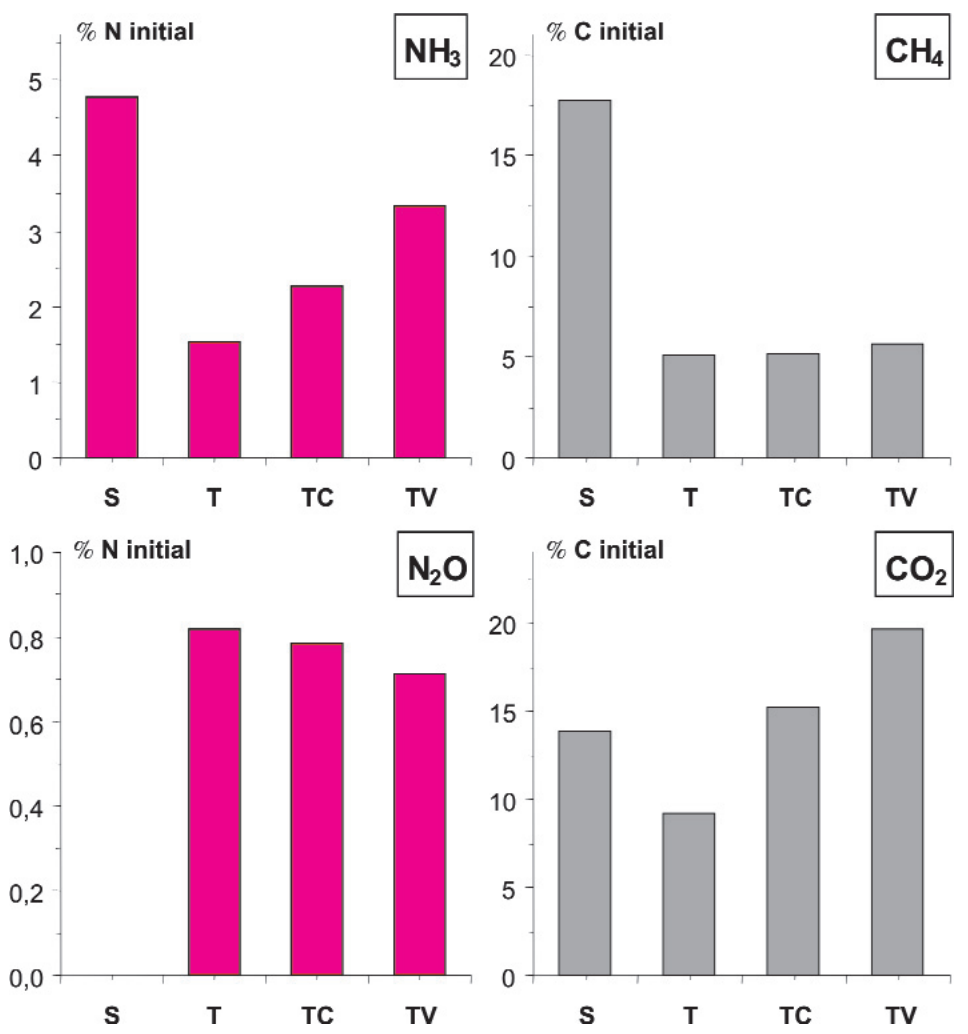

compte de la disponibilité de l'azote et la somme des fractions soluble et hémicelluloses dans la Matière Sèche (MS) obtenues par analyse selon Van Soest (1963), témoigne de la biodégradabilité du carbone. Les substrats de compostage à forte teneur en carbone biodégradable sont ainsi à rechercher pour limiter la volatilisation de $\mathrm{NH}_{3}$.

Dans un essai de longue durée en vraie grandeur, où du lisier en grande quantité relativement au substrat carboné (paille de blé) a été composté en couche assez mince $(40 \mathrm{~cm})$ dispersant la chaleur produite, l'émission de $\mathrm{NH}_{3}$ a été observée essentiellement pendant les phases d'apport de lisier et s'est limitée à $6 \%$ seulement de l'azote initial pour la période d'imprégnation-compostage qui dure $70 \mathrm{j}$, ce qui est bien moins qu'avec les techniques de compostage classiques en couches plus épaisses où l'élévation de température est plus forte. La mise en andain et la poursuite du compostage durant 4 mois ont provoqué une émission supplémentaire de $4 \%$ de l'azote initial. Dans la couche superficielle aérobie, la température modérée (40 à $45^{\circ} \mathrm{C}$ ) autorise le développement des flores bactériennes nitrifiantes. Par ailleurs, la forte disponibilité en $\mathrm{C}$ biodégradable favorise le développement de flores bactériennes immobilisant l'azote. Ces deux processus sont concurrents de la volatilisation. La couche inférieure en condition d'anoxie favorise quant à elle la dénitrification, en grande partie sous forme $\mathrm{N}_{2}$.

L'émission de $\mathrm{NH}_{3}$ provenant d'une litière de paille stockée en andain correspond à $7 \%$ de l'azote présent en début de stockage (Espagnol et al 2006), soit environ $3,5 \%$ de l'azote excrété par les animaux. L'aération périodique du fumier stocké a peu d'effet sur les quantités émises. Ce taux d'émission se situe dans la fourchette basse des valeurs rapportées dans la littérature, vraisemblablement en lien avec les températures relativement basses observées pendant la période de stockage. L'émission de $\mathrm{NH}_{3}$ est en effet assez sensible à la température externe, contrairement à celle des autres gaz dont les émissions sont dues à des processus internes aux andains.

\section{7 / Le traitement biologique du lisier permet de réduire les émis- sions de $\mathrm{NH}_{3}$, surtout en l'absen- ce de séparation de phases}

Par rapport au simple stockage en attente d'épandage, le traitement biologique aérobie du lisier conduit à une 
Figure 7. Répartition des émissions annuelles de gaz dans une station de traitement biologique de lisier de porc avec séparation de phase par centrifugation comportant un réacteur avec aérateur de surface (d'après Loyon et al 2006b, 2007).
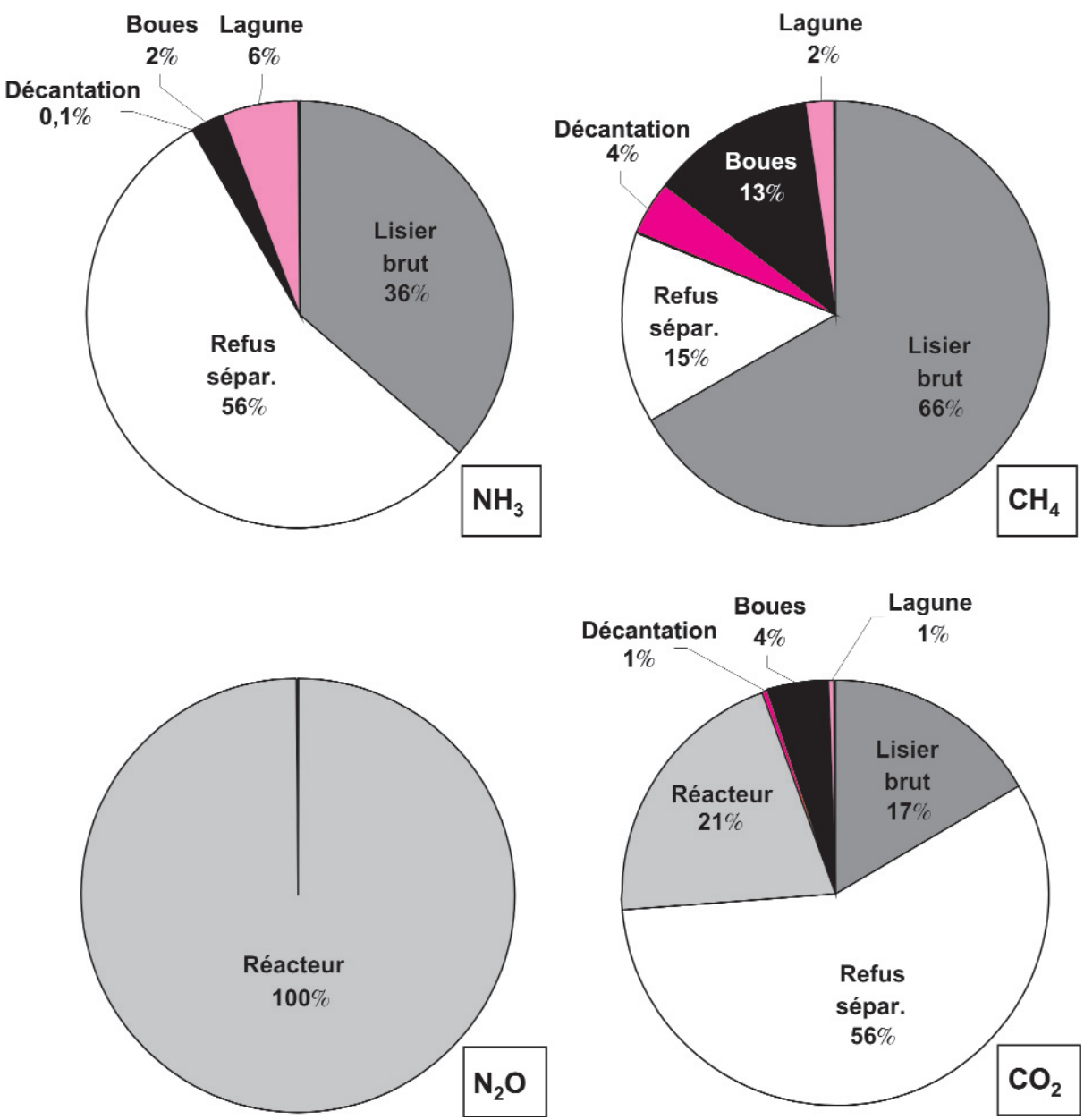

réduction sensible des émissions de $\mathrm{NH}_{3}$, évaluées respectivement à $4,8 \%$ à $1,5 \%$ de l'azote initialement présent dans le lisier (Loyon et al 2006b, 2007, figure 6). Pour un élevage naisseurengraisseur de 200 truies, les émissions de $\mathrm{NH}_{3}$ passent ainsi de 820 à $260 \mathrm{~kg}$ de $\mathrm{NH}_{3}-\mathrm{N}$ par an. Cette réduction est cependant moins importante lorsque qu'une séparation de phases est pratiquée préalablement au traitement, surtout dans le cas d'une séparation intensive avec une centrifugeuse. Les émissions de $\mathrm{NH}_{3}$ correspondent alors à plus des $2 / 3$ de celles observées en l'absence de traitement (soit 3,3\% de l'azote initialement présent dans le lisier). Loyon et al (2006b, 2007) ont pu établir la répartition des émissions de $\mathrm{NH}_{3}$ entre les différents modules d'une station de traitement utilisant une séparation de phases par centrifugation (figure 7). Ces émissions proviennent essentiellement des modules de stockage d'effluents non traités, à savoir la fosse d'homogénéisation du lisier brut et le stockage du refus solide de séparation. Aucune émission de $\mathrm{NH}_{3}$ n'est détectée au niveau du réacteur biolo-

On a longtemps considéré que le protoxyde d'azote $\left(\mathrm{N}_{2} \mathrm{O}\right)$ émis vers l'atmosphère provenait uniquement d'une dénitrification incomplète des formes oxydées de l'azote. Récemment, des travaux ont clairement montré que ce composé pouvait également être un coproduit des étapes de nitrification, aussi bien dans les sols que dans les systèmes d'épuration des effluents. Ces différentes étapes de nitrification/dénitrification nécessitent à la fois des conditions aérobies et anaérobies qui peuvent être réunies dans les litières, lors du compostage, dans les réacteurs de traitement biologique et dans le sol. Elles sont rarement réunies pendant les phases de simple stockage.

Dans le cas du traitement aérobie du lisier, des conditions défavorables (teneur en oxygène ou en carbone inadaptée, température du bassin d'aération trop élevée...) peuvent provoquer une inhibition partielle des processus de nitrification/dénitrification et être à l'origine de l'émission de $\mathrm{N}_{2} \mathrm{O}$. Dans le cas du compostage, la production de $\mathrm{N}_{2} \mathrm{O}$ pendant la nitrification pourrait être due à un manque relatif d'oxygène ne permettant pas une oxydation complète en nitrite puis nitrate. Ce phénomène intervient en début de compostage lorsque la température n'est pas encore très élevée (phase mésophile) permettant ainsi la nitrification et que le tas se sature rapidement en $\mathrm{CO}_{2}$ à cause de la dégradation rapide des composés carbonés facilement biodégradables. Pour la dénitrification, le manque de carbone biodégradable pourrait expliquer une réduction incomplète en $\mathrm{N}_{2}$.

\section{1 / Les émissions de $\mathrm{N}_{2} \mathrm{O}$ sont en général plus élevées avec de la litière que sur caillebotis, mais il y a de très fortes variations en fonction du mode de conduite de la litière}

Les émissions de $\mathrm{N}_{2} \mathrm{O}$ par les litières de sciure en couche fine sont assez importantes, représentant $12 \%$ de l'azote excrété par les animaux, dans les situations étudiées (Ramonet et Robin 2005). Ce résultat est à relier au fait que la couche fine est fortement remaniée par les animaux, faisant ainsi alterner les conditions aérobies, favorables à la nitrification, et anaérobies, propices à la dénitrification, avec un couplage des deux processus qui peut conduire à l'émission de $\mathrm{N}_{2} \mathrm{O}$, d'autant plus facilement que les températures atteintes ne sont pas très élevées.
Pour les systèmes litière couche profonde, Hassouna et al (2004) ont observés des émissions de $\mathrm{N}_{2} \mathrm{O}$ en station expérimentale et en élevages commerciaux entre 2 et $16 \%$ de l'azote excrété. En se basant sur ces résultats et sur la représentativité des résultats obtenus en 
Tableau 2. Facteurs d'émissions des gaz dans des bâtiments sur litière en comparaison avec des bâtiments sur caillebotis (d'après Ramonet et Robin 2002, CORPEN 2003, Robin et al 2004, Hassouna et al 2005).

\begin{tabular}{|c|c|c|c|}
\hline & $\begin{array}{c}\mathrm{NH}_{3} \\
(\% \mathrm{~N} \text { excrété) }\end{array}$ & $\begin{array}{c}\mathrm{N}_{2} \mathrm{O} \\
(\% \mathrm{~N} \text { excrété })\end{array}$ & $\begin{array}{c}\mathrm{CH}_{4} \\
\text { (kg/porc/an) }\end{array}$ \\
\hline Caillebotis & $15-30$ & $<5$ & 10 \\
\hline \multicolumn{4}{|l|}{ Litière } \\
\hline $\begin{array}{l}\text { - sciure couche fine } \\
\text { * mal maîtrisée } \\
\text { * bien maîtrisée }\end{array}$ & $\begin{array}{c}>60 \\
19\end{array}$ & 12 & \\
\hline $\begin{array}{l}\text { - paille couche épaisse } 50-80 \mathrm{~kg} / \text { porc } \\
\quad * \text { densité animale forte }\left(1,0-1,4 \mathrm{~m}^{2} / \text { porc }\right) \\
{ }^{*} \text { densité animale faible }\left(>2 \mathrm{~m}^{2} / \mathrm{porc}\right)\end{array}$ & $\begin{array}{l}15-25 \\
5-15\end{array}$ & $\begin{array}{c}4-12 \\
2-8\end{array}$ & $3-10$ \\
\hline - paille couche épaisse $\geq 100 \mathrm{~kg} /$ porc & 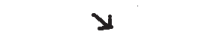 & $y$ & J \\
\hline
\end{tabular}

station expérimentale, Robin et al (2004) proposent un facteur d'émission pour le $\mathrm{N}_{2} \mathrm{O}$ compris entre 4 à $12 \%$ de l'azote excrété par les animaux pour les systèmes couche profonde avec un taux de paillage compris entre 50 et $80 \mathrm{~kg}$ paille/porc et une surface disponible de 1,0 à $1,4 \mathrm{~m}^{2}$ par porc (tableau 2). Toutes conditions égales par ailleurs, ces études ont montré qu'une réduction de la densité animale, au-delà de $2 \mathrm{~m}^{2}$ par porc, a tendance à réduire les émissions de $\mathrm{N}_{2} \mathrm{O}$ qui s'établissent alors entre 2 et $8 \%$ de l'azote excrété par les animaux. Ce résultat peut s'expliquer par le fait que la disponibilité relative de carbone augmente (moins de déjection par rapport à quantité de paille) ce qui favorise les fermentations bactériennes assurant à une réorganisation de l'azote. Le fait qu'une augmentation du paillage conduit aussi à une réduction des émissions de $\mathrm{N}_{2} \mathrm{O}$, est sans doute dû au même mécanisme. Les valeurs d'émissions de $\mathrm{N}_{2} \mathrm{O}$ observées sur litières sont toutes largement supérieures à celles observées dans les élevages sur caillebotis, inférieures à $0,2 \%$ selon l'IPCC (Intergovernmental Panel on Climate Change). De plus, la variabilité temporelle des émissions observées était de l'ordre de la variabilité entre élevage. Actuellement, les facteurs de variation ne sont pas encore complètement identifiés. Pour ce faire, et pour proposer des techniques permettant de réduire les émissions de $\mathrm{N}_{2} \mathrm{O}$ sans transfert de pollution, des études complémentaires doivent être menées.

2.2 / Le compostage en couche mince favorise les émissions de $\mathrm{N}_{2} \mathrm{O}$

Le compostage de déjections porcines amène à des émissions de $\mathrm{N}_{2} \mathrm{O}$ qui représentent environ $1 \%$ de l'azote présent dans les déjections (Paillat et al 2005a, figure 3). L'émission de $\mathrm{N}_{2} \mathrm{O}$ s'établit aussi rapidement que celle de
$\mathrm{NH}_{3}$ mais régresse très vite avec la montée en température du tas et l'émergence d'une flore thermophile. Cette émission du début de compostage correspond à la nitrification et à la dénitrification déjà en cours, dans le fumier notamment, et au début de nitrification dans la phase mésophile initiale et dans les parties du tas moins chaudes, en particulier en surface du tas. Elle apparaît à nouveau dans la phase de refroidissement, après $45 \mathrm{j}$. Cette reprise d'émission correspond à la dénitrification dans les agrégats et en fond de tas des nitrates produits.

Le compostage de lisier sur paille en couche mince de $40 \mathrm{~cm}$ environ conduit à des émissions de $\mathrm{N}_{2} \mathrm{O}$ représentant $3 \%$ de l'azote initial après $70 \mathrm{j}$ de compostage (Paillat et al 2005b), ce qui est notablement plus élevé que dans les techniques de compostage classiques. Ceci sous l'influence de deux facteurs. D'une part, la couche mince disperse la chaleur produite et favorise ainsi la flore nitrifiante. D'autre part, la quantipaille (rapport massique de 12:1) induit un fonctionnement anaérobie de la partie inférieure de la couche $(20-25 \mathrm{~cm})$. La nitrification est ainsi réalisée dans la couche supérieure $(15-20 \mathrm{~cm})$ et la dénitrification dans la couche inférieure et dans les agrégats. Ce fonctionnement en bi-couche permet les réactions de nitrification et de dénitrification simultanées conduisant à la production de $\mathrm{N}_{2} \mathrm{O}$ et surtout de $\mathrm{N}_{2}$ (41\% de l'azote initial). La mise en andain provoque une nouvelle émission de $\mathrm{N}_{2} \mathrm{O}$ qui atteint $6 \%$ de $\mathrm{N}$ initial pour une durée totale d'imprégnation et de compostage de 6 mois. La poursuite du compostage accentue aussi l'émission de $\mathrm{N}_{2}(44 \%$ de $\mathrm{N}$ initial).

En cohérence avec les résultats précédents, l'émission de $\mathrm{N}_{2} \mathrm{O}$ provenant té importante de lisier apportée sur la d'une litière de paille stockée en andain correspond à $3 \%$ de l'azote présent en début de stockage (Espagnol et al 2006), soit environ $1,5 \%$ de l'azote excrété par les animaux. L'aération périodique du fumier stocké a peu d'effet sur les quantités émises.

\section{3 / Les émissions de $\mathrm{N}_{2} \mathrm{O}$ lors du traitement biologique des lisiers dépendent de la technique d'aération utilisée}

Alors que le simple stockage du lisier brut ne conduit à aucune émission décelable de $\mathrm{N}_{2} \mathrm{O}$, le traitement biologique de ces lisiers, qui permet les réactions de nitrification et de dénitrification, conduit à des émissions que l'on peut estimer à $0,7-0,8 \%$ de l'azote initialement contenu dans les déjections (figure 6). La séparation de phase n'affecte guère les quantités de $\mathrm{N}_{2} \mathrm{O}$ produites qui proviennent exclusivement du réacteur biologique (figure 7), les modules de stockage, en amont et en aval du réacteur biologique n'en produisant pas de quantités détectables. Aucun des modules de stockage de la station de traitement n'offre les conditions pour une succession de conditions aérobies et anaérobies, nécessaire aux processus de nitrification/dénitrification à l'origine de la production de $\mathrm{N}_{2} \mathrm{O}$. Les températures élevées rencontrées dans le module de stockage des refus de séparation sont également défavorables à la production de $\mathrm{N}_{2} \mathrm{O}$ car elles inhibent le développement des bactéries nitrifiantes. Les quantités émises et l'origine de ces émissions dans le réacteur peuvent varier fortement. C'est ainsi que sur les trois stations suivies, on observe des variations d'un facteur 40 (Loyon et al 2006b). Les observations permettent de souligner la variabilité des émissions mais ne permettent pas de préciser les facteurs influençant ces variations (type d'aérateur, stratégie d'aération...). Pour l'estimation globale des émissions ci-dessus, le cas le plus défavorable a été pris en compte.

\section{4 / Les émissions de $\mathrm{N}_{2} \mathrm{O}$ dans le sol ne sont pas plus importan- tes après apports d'effluents ani- maux qu'après apports de ferti- lisants minéraux et restent difficiles à quantifier}

Les apports répétés de lisier ou de fumier composté de porc pendant une dizaine d'années sur une monoculture de maïs stimulent l'activité dénitrifiante potentiel du sol mais ne se sont traduits 
dans les deux situations étudiées, ni par une stimulation effective de la dénitrification mesurée à différentes dates à partir de sol en place, ni par une stimulation des émissions de $\mathrm{N}_{2} \mathrm{O}$ estimées sur l'ensemble d'une année, par comparaison aux mesures sur des sols soumis à une fertilisation azotée minérale.

Cette absence d'effet significatif sur la dénitrification mesurée sur le sol en place illustre le fait qu'une augmentation significative de la capacité de la microflore à dénitrifier ne se traduit pas obligatoirement par une augmentation effective de l'activité en conditions naturelles du fait de la régulation par un ou des facteurs limitants déterminants ; dans le cas présent le niveau d'humidité du sol et la teneur en nitrate ont été ces facteurs déterminants.

En ce qui concerne les flux de $\mathrm{N}_{2} \mathrm{O}$, malgré un niveau d'émission sensiblement plus faible à partir du sol recevant du lisier qu'à partir du sol soumis à une fertilisation minérale, les flux annuels estimés sous fertilisation organique et sous fertilisation minérale ne sont pas significativement différents (figure 8). Cette absence de différence est à mettre en relation avec la forte variabilité des mesures de ces émissions. Les cinétiques d'émission sont cependant modifiées par les apports de lisier ou de fumier composté, avec des flux de $\mathrm{N}_{2} \mathrm{O}$ plus importants dans la période qui suit les apports de produits organiques et moins importants le reste de l'année, comparés aux émissions sous apports de fertilisants minéraux. Des émissions significatives de $\mathrm{N}_{2} \mathrm{O}$ ont été notamment observées immédiatement après l'épandage de fumier composté en surface du sol, indiquant une émission directe à partir du compost épandu. La plus faible capacité d'émission de $\mathrm{N}_{2} \mathrm{O}$ en dehors des périodes suivant les apports d'effluents est attribuée à une augmentation de la capacité à réduire $\mathrm{N}_{2} \mathrm{O}$ en $\mathrm{N}_{2}$ liée aux apports organiques, qui se traduit par un abaissement du rapport $\mathrm{N}_{2} \mathrm{O} / \mathrm{N}_{2}$ émis au cours de la dénitrification en conditions anaérobies (Dambreville et al 2005, 2006a et b, 2008).

\section{3 / Les émissions de dioxyde de carbone}

Les émissions carbonées comprennent deux gaz à effet de serre, le dioxyde de carbone $\left(\mathrm{CO}_{2}\right)$ et le méthane $\left(\mathrm{CH}_{4}\right)$. Le $\mathrm{CO}_{2}$ issu de la biomasse n'est pas considéré comme polluant puisque la synthèse de la biomasse dont il est issu a soustrait la même quantité de gaz de l'atmosphère. Il n'en va pas de même pour le $\mathrm{CH}_{4}$ du fait que son potentiel «effet de serre» est très supérieur à celui du $\mathrm{CO}_{2}$ utilisé pour synthétiser la biomasse dont il est issu.

La répartition des émissions carbonées entre les deux gaz dépend pour une grande part de la teneur en oxygène de la matière organique en dégradation qui va conduire principalement à $\mathrm{du} \mathrm{CO}_{2}$ en conditions aérobies et à un mélange plus ou moins à part égale en conditions anaérobies.

\section{1 / Le compostage en couche mince conduit à des émissions de $\mathrm{CO}_{2}$ plus faibles}

Environ $40 \%$ du carbone initialement présent dans un compost est volatilisé

Figure 8. Estimation des émissions de $\mathrm{N}_{2} \mathrm{O}$ sur une année en utilisant différentes méthodes d'évaluation (d'après Dambreville et al 2006a, 2008, les lignes verticales représentent l'erreur standard).
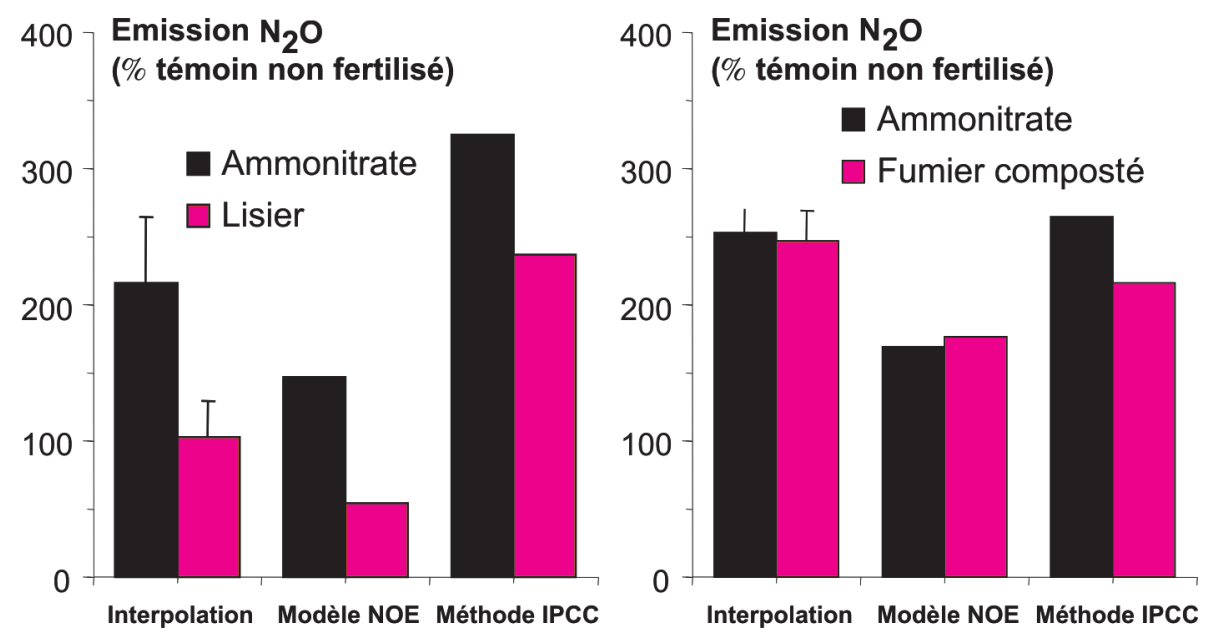

sous forme de $\mathrm{CO}_{2}$ (Paillat et al 2005a, figure 3) durant un compostage de deux mois. La variabilité des émissions en fonction des substrats est beaucoup plus faible que celle observée pour les émissions de gaz azotés, $\mathrm{NH}_{3}$ et $\mathrm{N}_{2} \mathrm{O}$. L'azote n'étant pas limitant dans les composts réalisés à partir de déjections animales, les émissions de $\mathrm{CO}_{2}$ peuvent être estimées à partir de la seule connaissance des quantités de carbone biodégradable.

Le compostage de lisier sur paille en couche mince $(40 \mathrm{~cm})$ conduit à des émissions de $\mathrm{CO}_{2}$ représentant $26 \%$ du carbone initial (Paillat et al 2005b) pendant la période d'imprégnationcompostage de $70 \mathrm{j}$, ce qui est sensiblement moins élevé qu'avec les techniques de compostage classiques. Ceci peut s'expliquer par le fait que la partie supérieure seulement de la couche (15$20 \mathrm{~cm}$ ) fonctionne en aérobiose, alors que la partie inférieure est majoritairement en anaérobiose avec des productions de chaleur et de $\mathrm{CO}_{2}$ réduites (la méthanisation des sucres produit 7 fois moins de chaleur et 2 fois moins de $\mathrm{CO}_{2}$ que l'oxydation). La mise en andain et la poursuite du compostage durant 4 mois permet la poursuite de la dégradation de la Matière Organique (MO), avec une production de $\mathrm{CO}_{2}$ qui atteint alors $57 \%$ du carbone initial.

Les émissions de $\mathrm{CO}_{2}$ provenant d'une litière de paille stockée en andain correspondent à $11 \%$ du carbone présent en début de stockage (Espagnol et al 2006), ce qui montre le peu de transformation de la MO lors d'un simple stockage, relativement au compostage.

\section{2 / Le traitement biologique du lisier conduit à des émissions de $\mathrm{CO}_{2}$ plus faibles que le sim- ple stockage du lisier, à condi- tion qu'il n'y ait pas de sépara- tion de phases}

Le traitement biologique des lisiers conduit à des émissions de $\mathrm{CO}_{2}$ sensiblement identiques à celles observées lors du simple stockage du lisier avant épandage ( 9 à $20 \%$ suivant la filière de traitement contre $14 \%$ du carbone initialement présent dans les déjections, figure 6). Lorsque le traitement est précédé d'une séparation de phases, le module de stockage des refus de séparation est le contributeur majeur à l'émission de $\mathrm{CO}_{2}$ (figure 7). Les quantités émises par le réacteur biologique varient fortement en fonction du système d'aération (Loyon et al 2006a). 


\section{4 / Les émissions de mé- thane}

La production de méthane $\left(\mathrm{CH}_{4}\right)$ est possible dans les conditions d'anoxie qui peuvent prendre place dans les litières très humides, au fond des tas lors du stockage ou du compostage et dans le stockage et le traitement biologique des effluents liquides tels que le lisier. Il correspond à la dégradation des composés carbonés facilement biodégradables, en particulier les sucres, en $\mathrm{CH}_{4}$ et $\mathrm{CO}_{2}$ avec une faible production de chaleur (7 fois inférieure à la chaleur produite par oxydation des sucres). Son émission est dépendante du transfert au sein des masses solide ou liquide. En effet, dans le cas des produits solides au stockage et en compostage, une grande part du $\mathrm{CH}_{4}$ produit dans les zones anoxiques peut être oxydée dans les couches aérobies de surface.

\section{1 / Les émissions de $\mathrm{CH}_{4}$ sont plus faibles sur litière que sur caillebotis}

En bâtiment, Hassouna et al (2005) ont établi que les émissions de $\mathrm{CH}_{4}$ des élevages sur litière sont inférieures à celles des systèmes caillebotis $(10 \mathrm{~kg}$ $\mathrm{CH}_{4}$ /porc.an ; IPCC 2003). Cependant une grande variabilité à la fois temporelle et entre élevage a été observée. Les émissions les plus importantes ont été observées dans un élevage présentant une litière très humide et donc des conditions favorables aux réactions anaérobiques à l'origine de l'apparition du méthane.

\section{2 / Les émissions de $\mathrm{CH}_{4}$ sont faibles lors du compostage, sauf s'il est réalisé en couche mince}

Les émissions de $\mathrm{CH}_{4}$ lors du compostage sont faibles. Elles représentent moins de $0,1 \%$ du carbone initial (Paillat et al 2005a, figure 3). La dynamique d'émission du $\mathrm{CH}_{4}$ est similaire à celle de $\mathrm{NH}_{3}$, ces 2 gaz étant émis principalement pendant la phase thermophile. Le $\mathrm{CH}_{4}$ trouve son origine dans les fermentations microbiennes des zones anaérobies du compost, mais la plus grande part est oxydée dans les couches superficielles riches en oxygène.

Le compostage de lisier sur paille en couche mince $(40 \mathrm{~cm})$ conduit à des émissions de $\mathrm{CH}_{4}$ représentant $3 \%$ du carbone initial (Paillat et al 2005b) durant la phase d'imprégnationcompostage, ce qui est nettement plus élevé qu'avec les techniques de compostage classiques. Les épandages successifs de lisier riche en MS (6\%) apportant de manière répétée beaucoup de carbone très biodégradable, l'importance de la couche saturée en lisier (20$25 \mathrm{~cm}$ ) relativement à la couche aérobie $(15-20 \mathrm{~cm})$ et l'augmentation de température (environ $40^{\circ} \mathrm{C}$ ) expliquent d'une part la forte production de méthane dans la couche anaéorobie d'autre part et sa faible oxydation lorsqu'il traverse la couche aérobie. Après la mise en andain et lors de la maturation du compost durant 4 mois, la dégradation de la $\mathrm{MO}$ se poursuit et conduit à $3 \%$ supplémentaire du carbone initial émis sous forme de méthane. Cette production est liée à la présence persistante de carbone biodégradable et au produit relativement humide issu de la phase d'imprégnation-compostage.

Les émissions de $\mathrm{CH}_{4}$ provenant d'une litière de paille stockée en andain sont quasi nulles lorsque le fumier est périodiquement retourné (Espagnol et al 2006) et un peu plus élevées lorsqu'il ne l'est pas ( $0,8 \%$ du C initialement présent).

\section{3 / Le traitement biologique du lisier conduit à une forte réduction des émissions de $\mathrm{CH}_{4}$ par rapport à un simple stockage}

Le traitement biologique des lisiers conduit à une réduction drastique des émissions de $\mathrm{CH}_{4}$ par rapport au simple stockage du lisier avant épandage (5$6 \%$ contre $18 \%$ du carbone initialement présent dans les déjections). Ces émissions proviennent pour $70 \%$ de la fosse de stockage du lisier brut, le reste étant réparti entre les autres modules (figure 7). La part du réacteur biologique est faible, moins de $1 \%$ du carbone initial du lisier.

\section{5 / Synthèse des connaissan- ces acquises sur les émis- sions de $\mathrm{NH}_{3}$ et de $\mathrm{N}_{2} \mathrm{O}$ tout au long de la filière de pro- duction}

Les figures 9 à 13 présentent une tentative de synthèse des connaissances acquises sur les émissions de $\mathrm{NH}_{3}$ et de
$\mathrm{N}_{2} \mathrm{O}$. Elles tentent de quantifier les différentes voies d'émissions de composés azotés gazeux aux divers étages des filières considérées :

- filières lisier, avec ou sans compostage (figure 9) ;

- filières lisier avec traitement biologique, comprenant ou non un module de séparation de phases (figure 10) ;

- filières litière de paille épandue directement ou après compostage (figure 11) ;

- filières litière de sciure épandue directement ou après compostage (figure 12).

La figure 13 représente le cumul des émissions de composés azotés polluants dans les différentes filières. L'utilisation de litière conduit à des émissions significatives de $\mathrm{N}_{2} \mathrm{O}$ avant épandage, qui représentent environ $10 \%$ de l'azote excrété par les animaux. Leur impact «effet de serre» est donc plus important que dans les filières qui n'en utilisent pas, où les émissions de $\mathrm{N}_{2} \mathrm{O}$ sont de l'ordre de $1 \%$ de l'azote excrété. Les filières avec litière tendent par contre à émettre moins de $\mathrm{NH}_{3}$. Le compostage avec un substrat de paille entraîne une augmentation notoire des émissions de $\mathrm{NH}_{3}$, qu'il s'agisse de compostage de litière ou de lisier. Les émissions de $\mathrm{N}_{2} \mathrm{O}$ au champ sont globalement faibles en regard de celles intervenant avant épandage. Elles sont extrêmement faibles lorsque l'azote arrive au sol sous forme essentiellement minérale. Elles sont plus élevées, de l'ordre de 0,10 $0,15 \%$ de l'azote excrété par an, lorsque l'azote arrive au sol sous forme organisée, dans les filières impliquant une litière et/ou un compostage.

Quelles que soient les filières, le poste d'émissions de $\mathrm{NH}_{3}$ le plus important est le bâtiment d'élevage proprement dit où entre 20\% (animaux sur litières) et 25\% (animaux sur caillebotis) de l'azote excrété est émise sous cette forme. En ce sens, l'évacuation pluriquotidienne des effluents (Callarec et al 2005) est une voie intéressante de réduction des émissions. Les concentrations excessives de $\mathrm{NH}_{3}$ dans l'air ambiant ont en effet une incidence défavorable sur la santé des animaux et des travailleurs. L'épandage de lisier liquide ou le compostage de substrats pailleux conduisent aussi à des émissions importantes de $\mathrm{NH}_{3}$. 
Figure 9. Emissions de $\mathrm{NH}_{3}$ et de $\mathrm{N}_{2} \mathrm{O}$ dans le cas d'une filière «lisier» avec épandage sans traitement ou compostage. Les émissions sont exprimées en g par kg d'azote excrété par les animaux (valeurs en gras) ou en \% de l'azote entrant dans le compartiment. Les chiffres en noir correspondent aux données de CORPEN (2003). Les chiffres en rouge correspondent aux données acquises dans le cadre du programme «Porcherie verte».

Compostage de lisier

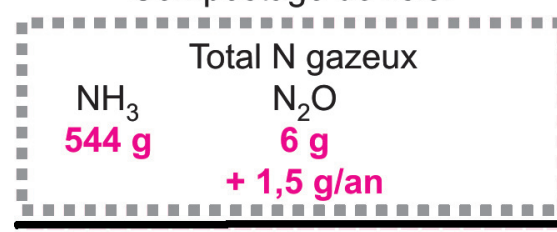

Epandage de lisier

$\begin{array}{cc} & \text { Total } \mathrm{N} \text { gazeux } \\ \mathrm{NH}_{3} & \mathrm{~N}_{2} \mathrm{O} \\ \mathbf{4 3 0} \mathbf{g} & \\ & 0,1 \mathrm{~g} / \mathrm{an}\end{array}$

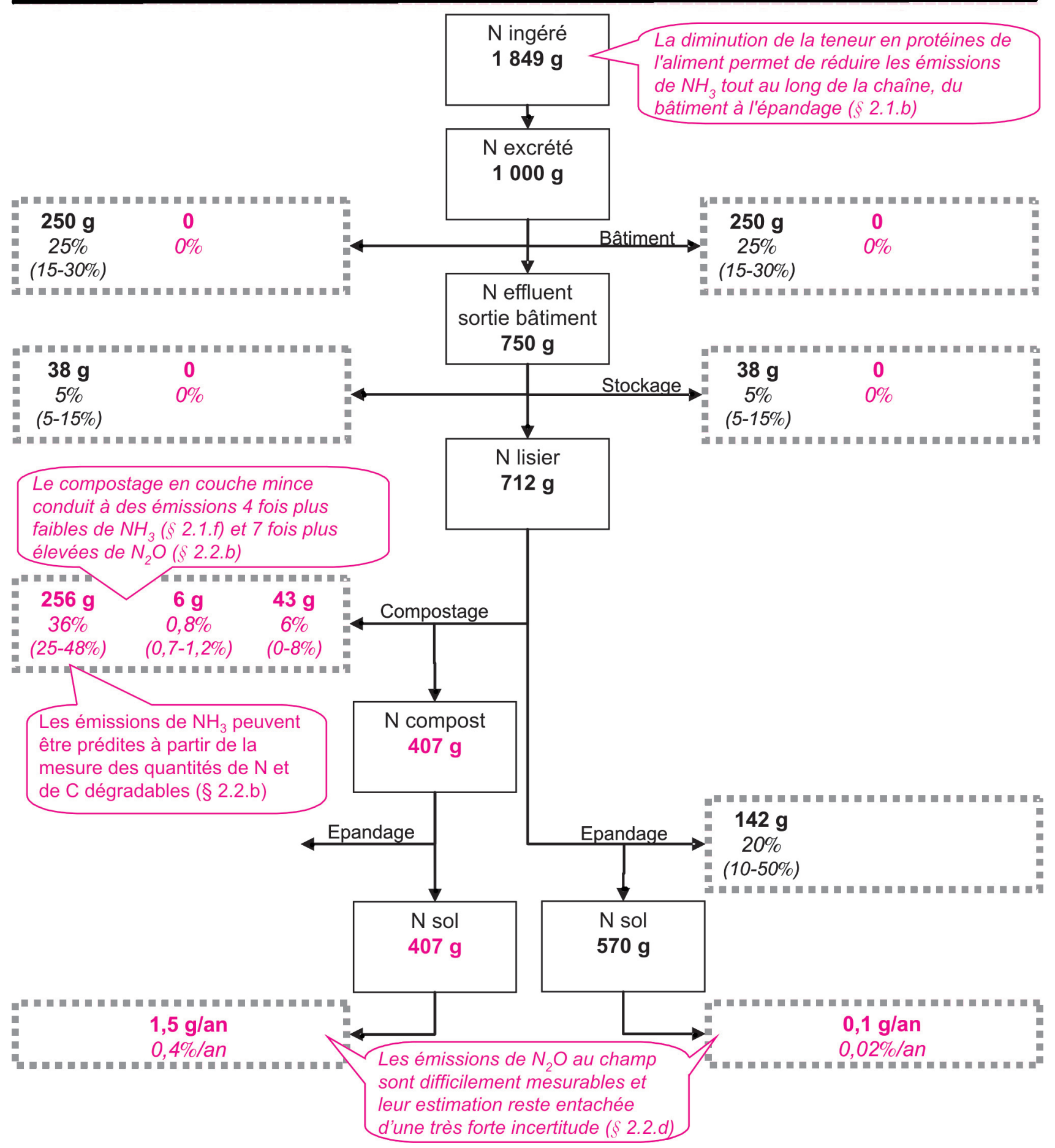


Figure 10. Emissions de $\mathrm{NH}_{3}$ et de $\mathrm{N}_{2} \mathrm{O}$ dans le cas d'une filière «lisier» avec traitement biologique. Les émissions sont exprimées en g par kg d'azote excrété par les animaux (valeurs en gras) ou en \% de l'azote entrant dans le compartiment. Les chiffres en noir correspondent aux données de CORPEN (2003). Les chiffres en rouge correspondent aux données acquises dans le cadre du programme «Porcherie verte».

Sans séparation de phase

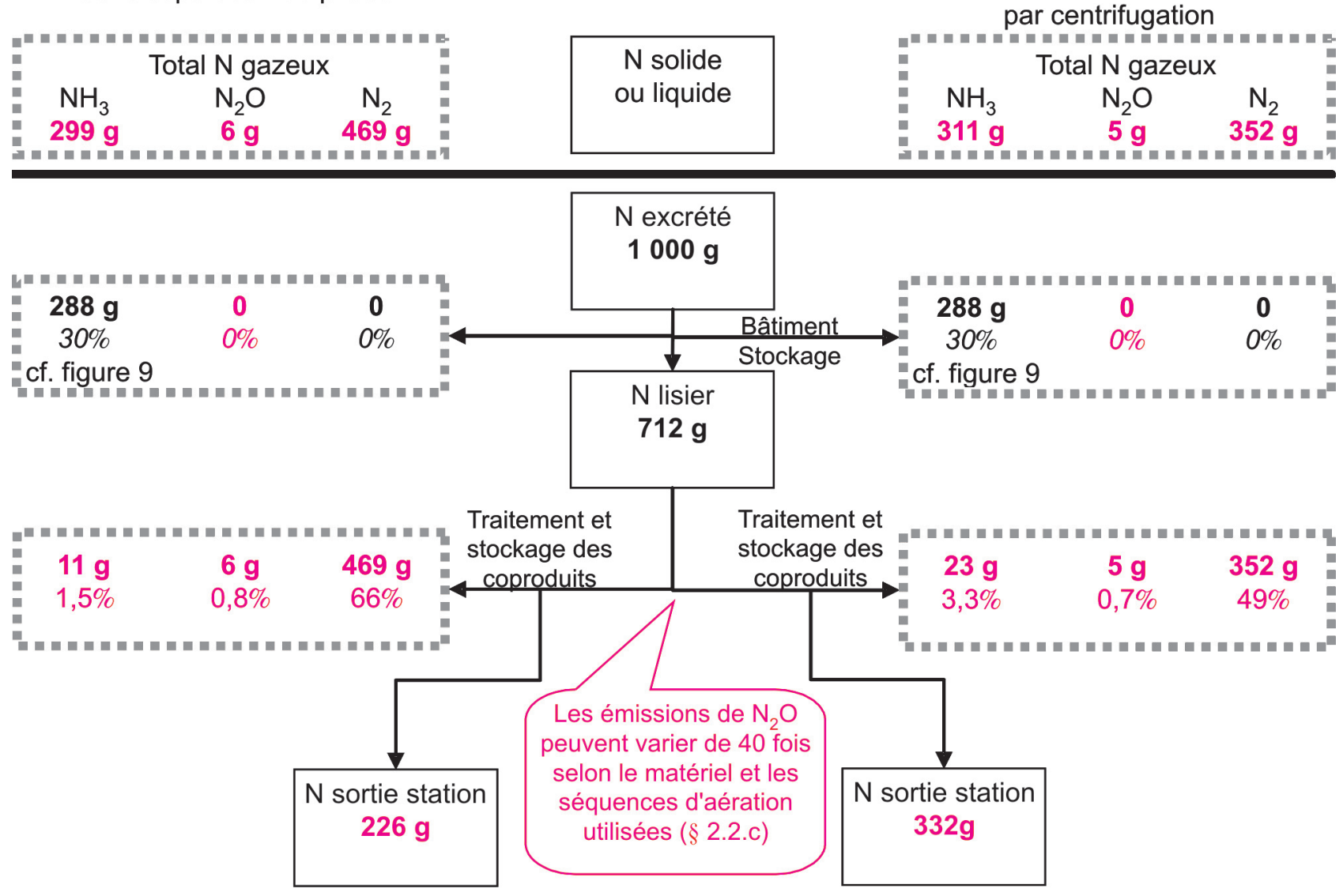

\section{Conclusions}

Dans le prolongement des travaux antérieurs, dont les résultats sont résumés dans CORPEN (2003), les recherches conduites dans le cadre du programme «Porcherie verte» ont apporté des éléments de quantification des émissions gazeuses, tout particulièrement pour les filières utilisant le traitement biologique du lisier, les litières de paille ou le compostage (lisier ou litière). L'apport d'information a été particulièrement important pour les émissions de $\mathrm{N}_{2} \mathrm{O}$ qui étaient auparavant fort mal connues. Les facteurs de maîtrise des émissions de $\mathrm{NH}_{3}$ sont maintenant raisonnablement bien connus. Il n'en va pas de même pour les émissions de $\mathrm{N}_{2} \mathrm{O}$ qui peuvent varier dans des proportions extrêmement importantes en fonction des modes de conduite des litières, des méthodes de compostage ou des conditions de température et d'humidité des sols. Les incertitudes sur les émissions de $\mathrm{N}_{2} \mathrm{O}$ restent encore très élevées.
Les litières et le compostage ont une excellente image de marque auprès du grand public et sont souvent réputées avoir beaucoup moins d'impact sur l'environnement que le lisier. Cette croyance résulte pour beaucoup du fait que ces techniques sont effectivement très efficaces pour diminuer les nuisances olfactives (cf. article sur les odeurs). Les résultats obtenus par le programme «Porcherie verte» conduisent à nuancer ce point de vue :

- les filières litières émettent en général moins de $\mathrm{NH}_{3}$ que les filières lisier (à condition de ne pas composter le fumier de paille), mais elles émettent beaucoup plus de $\mathrm{N}_{2} \mathrm{O}$, qui a un potentiel effet de serre 300 fois supérieur à celui du $\mathrm{CO}_{2}$;

- le compostage du lisier entraîne une augmentation des émissions gazeuses ;

- litières et compostage volatilisent une part importante de l'azote des effluents, gaspillant ainsi un potentiel fertilisant important qu'il faut remplacer par des engrais minéraux qui coûtent cher en énergie aussi bien pour leur production que pour leur transport.
Or la production d'énergie a un très fort impact sur l'effet de serre ;

- la recherche d'économie sur le coût des litières ou du compostage, en réduisant la quantité de substrat carboné apporté par rapport aux quantités de déjections émises, conduit à aggraver les transferts de pollution vers l'atmosphère. C'est ainsi que l'utilisation de couches fines de litière ou l'augmentation de la densité d'animaux accélèrent les émissions de $\mathrm{NH}_{3}$ et de $\mathrm{N}_{2} \mathrm{O}$. De même, le compostage en couche mince réduit les émissions de $\mathrm{NH}_{3}$ mais accroît énormément celles de $\mathrm{N}_{2} \mathrm{O}$.

Ces constations ne remettent bien entendu pas en cause les effets bénéfiques des litières sur la réduction des nuisances olfactives et l'amélioration du bien-être des animaux.

Le traitement biologique du lisier semble à première vue avoir un impact favorable puisqu'il conduit à réduire les émissions de $\mathrm{NH}_{3}$ sans trop relarguer de $\mathrm{N}_{2} \mathrm{O}$. Mais c'est oublier que le traitement biologique coûte cher en énergie et qu'il détruit l'essentiel de la 
Figure 11. Emissions de $\mathrm{NH}_{3}$ et de $\mathrm{N}_{2} \mathrm{O}$ dans le cas de filières «litières» utilisant un substrat de paille. Les émissions sont exprimées en g par kg d'azote excrété par les animaux (valeurs en gras) ou en \% de l'azote entrant dans le compartiment. Les chiffres en noir correspondent aux données de CORPEN (2003). Les chiffres en rouge correspondent aux données acquises dans le cadre du programme «Porcherie verte».

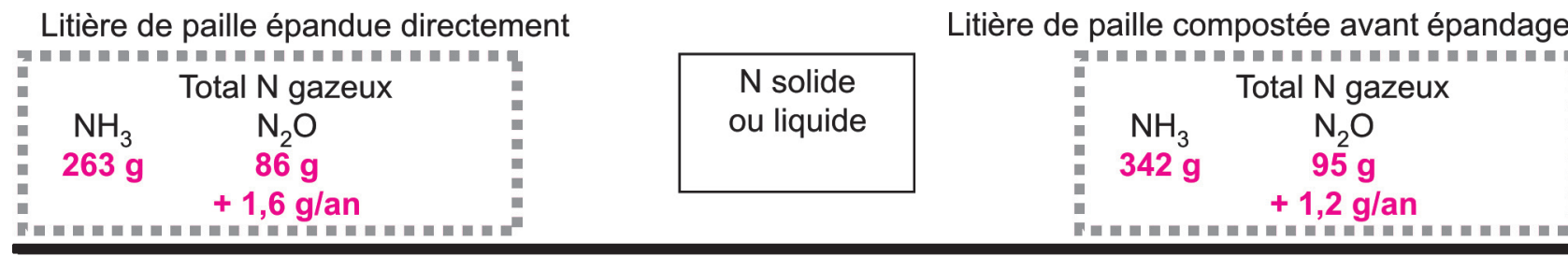

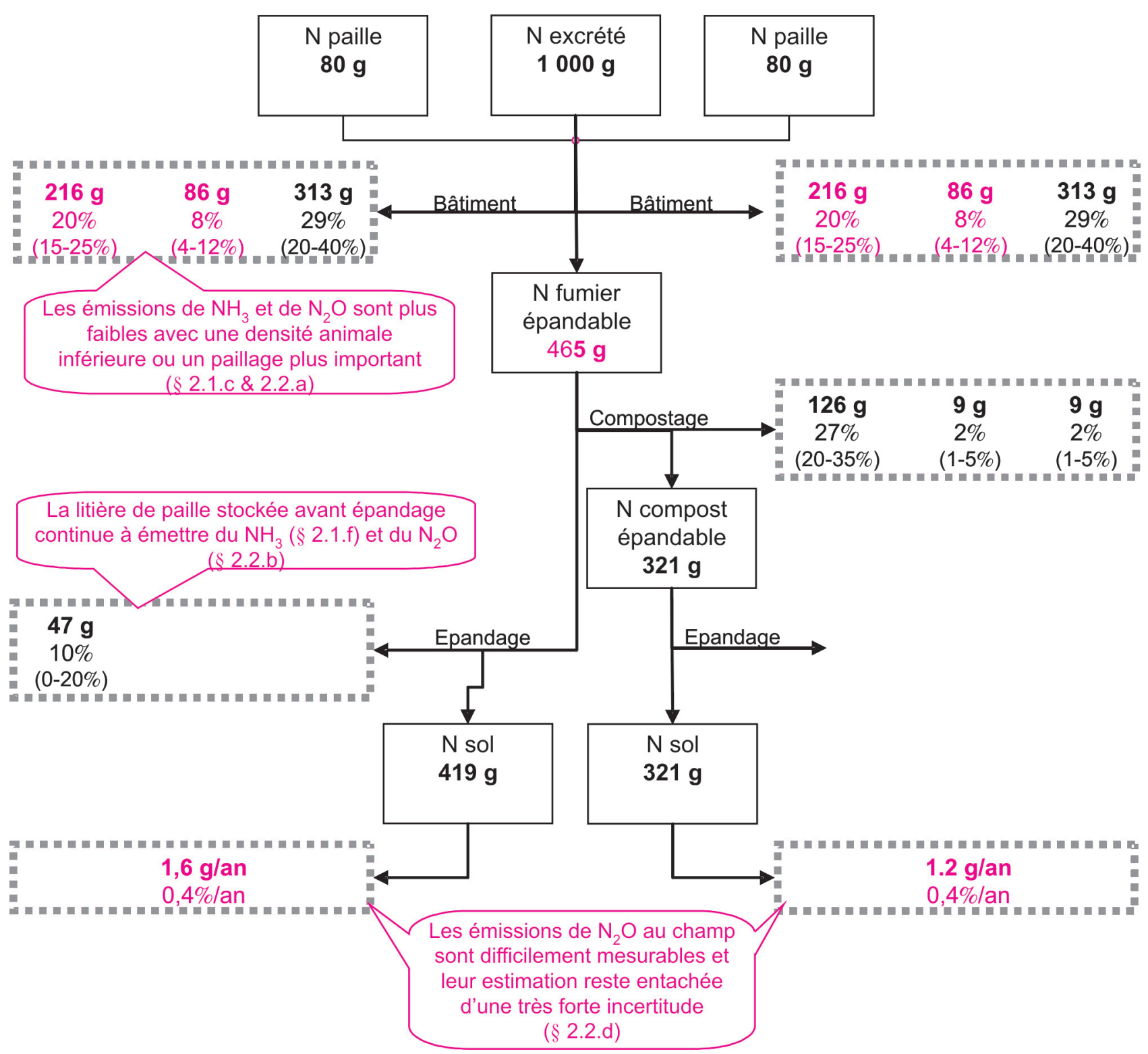

valeur fertilisante azotée des effluents, ce qui revient à un gaspillage d'énergie (voir plus haut). Ces deux effets conjugués contribuent fortement à l'effet de serre, même si c'est de façon décentralisée, en dehors de l'exploitation. Comme pour la litière ou le compos- tage, on a là un double transfert de pollution, du sol et de l'eau vers l'atmosphère d'une part, et de l'exploitation vers l'extérieur de l'exploitation d'autre part. Le transfert géographique des émissions de l'exploitation vers l'extérieur ne change bien évidemment rien à l'impact «effet de serre». Ce point souligne l'importance des approches globales que nous développons dans un autre article de ce numéro. 
Figure 12. Emissions de $\mathrm{NH}_{3}$ et de $\mathrm{N}_{2} \mathrm{O}$ dans le cas de filières «itières» utilisant un substrat de sciure de bois. Les émissions sont exprimées en g par kg d'azote excrété par les animaux (valeurs en gras) ou en \% de l'azote entrant dans le compartiment. Les chiffres en noir correspondent aux données de CORPEN (2003). Les chiffres en rouge correspondent aux données acquises dans le cadre du programme «Porcherie verte».

Litière de sciure épandue directement

Litière de sciure compostée avant épandage

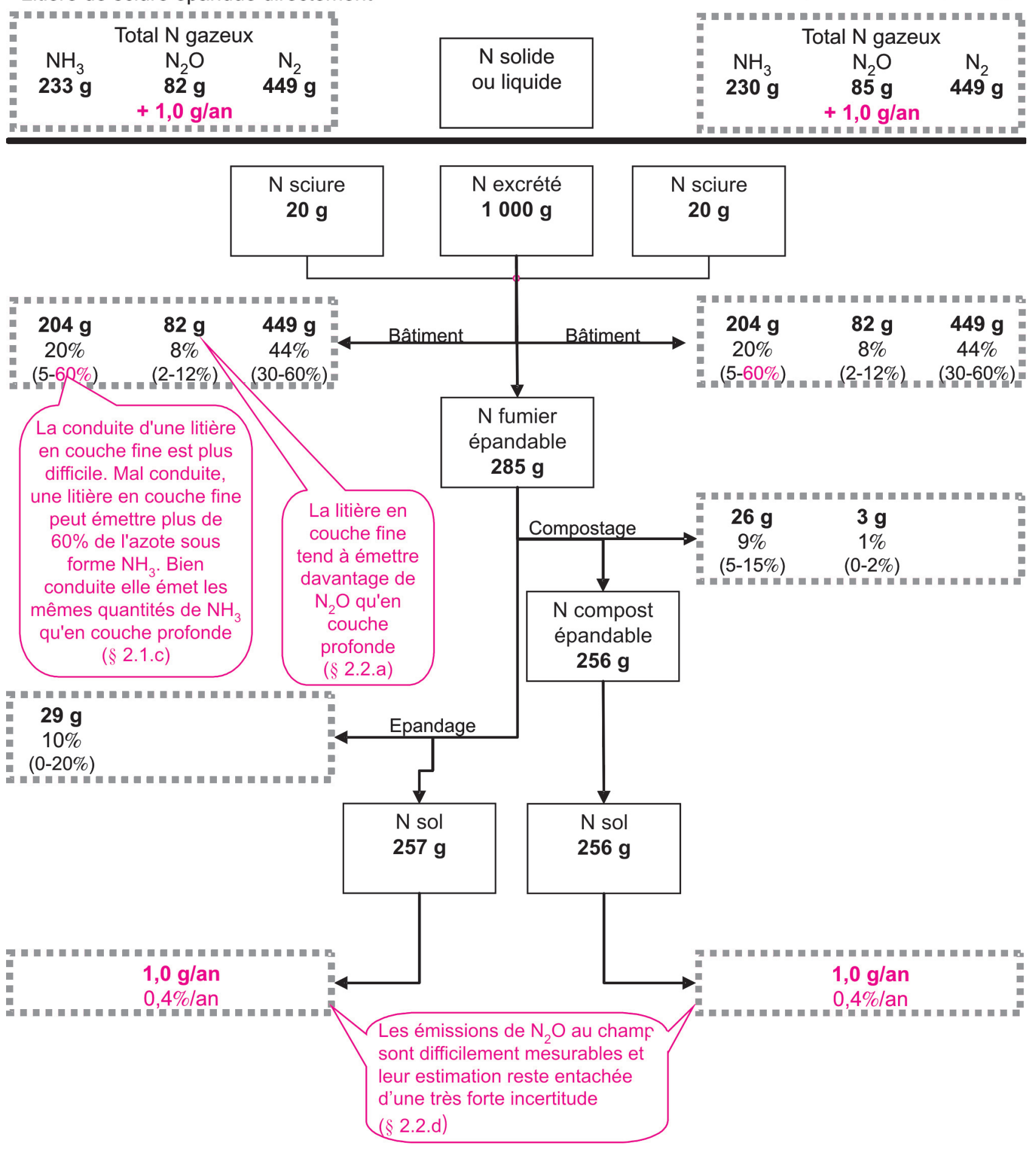


Figure 13. Emissions totales de $\mathrm{NH}_{3}$ et de $\mathrm{N}_{2} \mathrm{O}$ dans différentes filières de gestion des effluents porcins. Les émissions avant épandage (barres horizontales) sont exprimées en \% de l'azote excrété par les animaux. Les émissions de $\mathrm{N}_{2} \mathrm{O}$ au champ (courbe rouge) sont exprimées par an, en \% de l'azote excrété par les animaux.

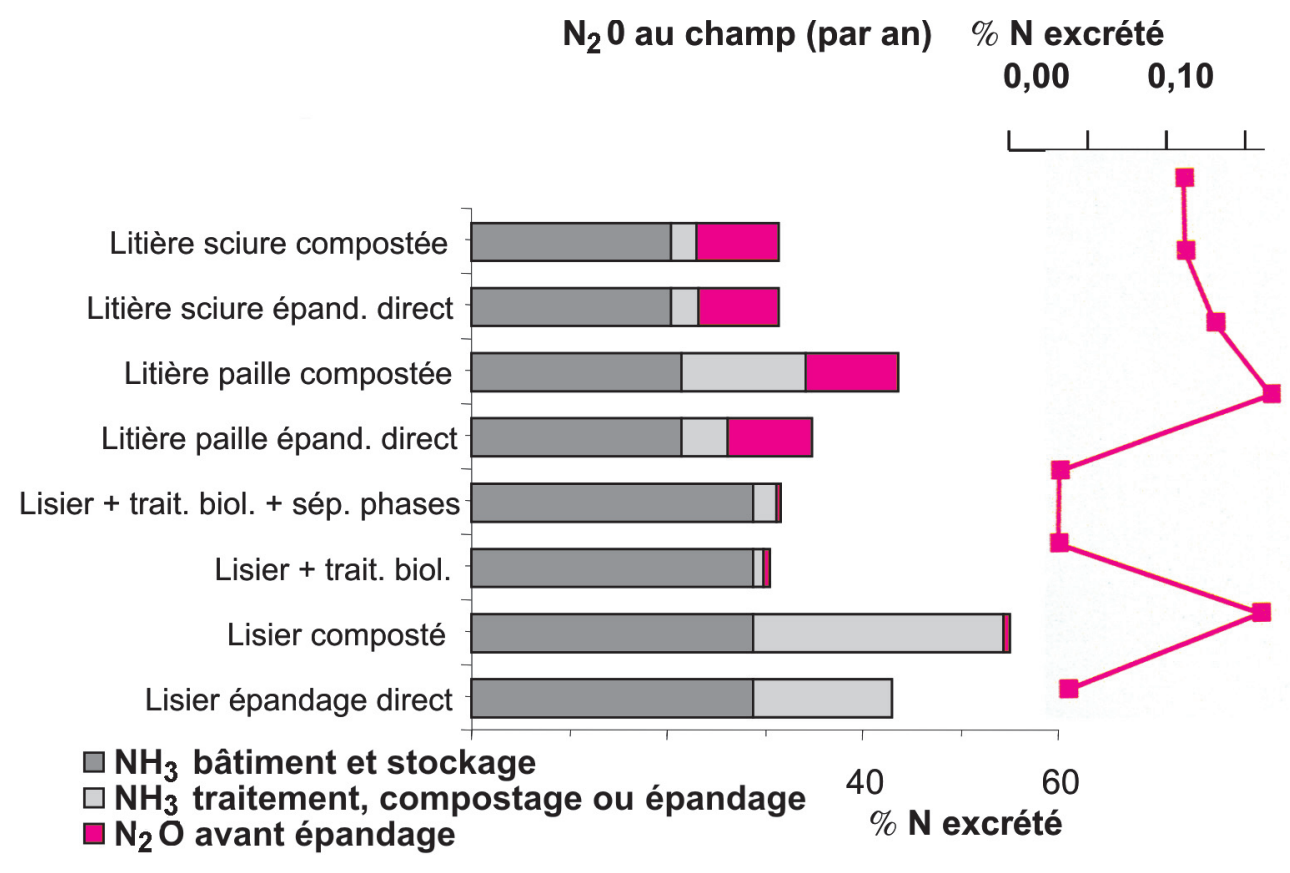

\section{Références}

Callarec J., Robin P., Bouché M., Morvan T. 2005. Rapid removal of pig slurry and technical feasibility of recycling treated flushed liquid. Int. Workshop on green pork production, May 25-27, Paris, France, 109-110.

CORPEN, 2003. Estimation des rejets d'azote - phosphore - potassium - cuivre et zinc des porcs. MAAPAR - MEDD, 44p.

Dambreville C., Hénault C., Philippot L., Bizouard F., Morvan T., Chaussod R., Germon J.C., 2006a. Compared effects of long-term pig slurry applications and mineral fertilization on soil denitrification and its end products $\left(\mathrm{N}_{2} \mathrm{O}\right.$, $\mathrm{N}_{2}$ ). Biol. Fertil. Soils, 42, 490-500.

Dambreville C., Hallet T.S., Nguyen C., Morvan T., Germon J.C., Philippot L., 2006b. Structure and activity of the denitrifying community in a maize-cropped field fertilized with composted pig manure or ammonium nitrate. FEMS Microbiol. Ecol., 56, 119-131.

Dambreville C., Morvan T., Germon J.C. 2008. $\mathrm{N}_{2} \mathrm{O}$ emission in maize-crops fertilized with pig slurry, matured pig manure or ammonium nitrate in Brittany. Agric., Ecosyst. Env., 123, 201-210.

Dourmad J.Y., Pomar C., Massé D., 2002. Modélisation du flux de composés à risque pour l'environnement dans un élevage porcin. Journ. Rech. Porcine Fr., 34, 183-194.

Espagnol S., Hassouna M., Robin P., Levasseur P., Paillat J.M., 2006. Emissions gazeuses de $\mathrm{NH}_{3}, \mathrm{~N}_{2} \mathrm{O}, \mathrm{CH}_{4}$ lors du stockage de fumier de porc provenant d'une litière accumulée : effets du retournement. Journ. Rech. Porcine Fr., 38, 41-48.

Hassouna M., Robin P., Texier C., 2004. $\mathrm{N}_{2} \mathrm{O}$ and $\mathrm{CH}_{4}$ emissions in French straw-based pigon-litter breeding systems. Int. Conf. on green- house gas emissions from agriculture - mitigation options and strategies, 10-12 February, Leipzig, Allemagne, 245-247.

Hassouna M., Robin P., Texier C., 2005. $\mathrm{NH}_{3}$, $\mathrm{N}_{2} \mathrm{O}$ and $\mathrm{CH}_{4}$ emission factors from pig-on-litter systems. Int. workshop on green pork production, May 25-27, Paris, France, 121-122.

IPPC, 2003. http://www.ipcc-nggip.iges.or.jp/ EFDB/find_ef.php, 9 décembre 2003.

Kermarrec C., Robin P., 2002. Emissions de gaz azotés en élevage de porcs sur litière de sciure. Journ. Rech. Porcine Fr., 34, 155-160.

Lebret B., Couvreur S., Meunier-Salaün M., Guingand N., Robin P., Hassouna M., Cariolet R., Dourmad J.Y., 2004. Comparaison expérimentale de deux conduites d'élevage de porcs en croissance. Journ. Rech. Porcine Fr., 36, 53-62.

Loyon L., Peu P., Guiziou F., Picard S., 2006a. Emissions gazeuses de $\mathrm{NH}_{3}, \mathrm{~N}_{2} \mathrm{O}, \mathrm{CH}_{4}, \mathrm{CO}_{2}$ au cours du traitement biologique du lisier de porc selon trois modes d'aération, Journ. Rech. Porcine Fr., 38, 49-52.

Loyon L, Guiziou F, Beline F, Peu P, 2006b. Gaseous emissions $\left(\mathrm{NH}_{3}, \mathrm{~N}_{2} \mathrm{O}, \mathrm{CH}_{4}\right.$ and $\left.\mathrm{CO}_{2}\right)$ during pig slurry biological aerobic treatment and treatment by-product storages. Int. Congr., Series 1293, 299-302.

Loyon L., Guiziou F., Beline F., Peu P, 2007. Gaseous Emissions $\left(\mathrm{NH}_{3}, \mathrm{~N}_{2} \mathrm{O}, \mathrm{CH}_{4}\right.$ and $\left.\mathrm{CO}_{2}\right)$ from the aerobic treatment of piggery slurry. Comparison with a conventional storage system. Biosyst. Eng., 97, 472-480.

Paillat J.M., Robin P., Hassouna M., Leterme P., 2005a. Predicting ammonia and carbon dioxide emissions from carbon and nitrogen biodegradability during animal waste composting. Atmospheric Env., 39, 6833-6842.
Paillat J.M., Robin P., Hassouna M., Callarec J., Toularastel P., 2005b. Environmental assessment of composting pig slurry with wheat straw based on the Guernevez ${ }^{\circledR}$ process. Int Workshop on green pork production, May 25-27, Paris, France. 110p.

Portejoie S., 2002. Volatilisation ammoniacale lors du stockage et à l'épandage de lisiers de porc : impact des apports protéiques, de l'utilisation. Thèse de doctorat, Université de Perpignan, France, 137p

Portejoie S., Dourmad J.Y., Martinez J., Lebreton Y., 2002. Effet de la réduction du taux protéique de l'aliment sur la volatilisation ammoniacale des effluents porcins. Journ. Rech. Porcine Fr., 34, 167-174.

Portejoie S., Dourmad J.Y., Martinez J., Lebreton Y., 2004. Effect of lowering dietary crude protein on nitrogen excretion, effluent composition and ammonia emission from fattening pigs. Livest. Prod. Sci., 91, 45-55.

Ramonet Y., Robin P., 2002. L'engraissement de porcs sur litière de particules de bois ou de sciure en couche fine. Journ. Rech. Porcine Fr., 34, 143-148.

Ramonet Y., Robin P., 2005. Nitrogen and phosphorus balance for fattening pigs on shallow litter. Int. Workshop on green pork production, May 25-27, Paris, France, 139-140.

Ramonet Y., Guivarch C., Dappelo C., Robin P., Laplanche A., Prado N., Amrane A. Meinhold J., Ochoa J.C., Li Y., Callarec J., 2007. Le lisier frais : évacuation fréquente des lisiers des porcheries. Faisabilité technique et conséquences environnementales. Journ. Rech. Porcine Fr., 36, 31-42.

Robin P., Hassouna M., Texier C., 2004. Emissions d'ammoniac et de protoxyde d'azote 
des porcs engraissés sur litière de paille. Journ. Rech. Porcine Fr., 36, 63-70.

Thirion F., Béline F., Saint Cast P., 2005. Liquid manure mixing and ammonia emissions.
Int. Workshop on green pork production, May 25-27, Paris, France, 141-142.

Van-Soest P.J., 1963. Use of detergents in the analysis of fibrous feeds. II. A rapid method for the determination of fibre and lignin. J. Ass. Anal. Chemist., 46, 829-835.

\section{Résumé}

Cet article résume les apports du programme «Porcherie verte» dans la connaissance et la maîtrise des émissions gazeuses intervenant lors des différentes phases de l'élevage porcin. Ces émissions comprennent des gaz à effet de serre, particulièrement le méthane $\left(\mathrm{CH}_{4}\right)$ et le protoxyde d'azote $\left(\mathrm{N}_{2} \mathrm{O}\right)$, ainsi que l'ammoniac $\left(\mathrm{NH}_{3}\right)$, nocif pour les animaux et pour l'Homme et qui a un impact sur l'environnement en termes d'acidification et d'eutrophisation.

Les émissions de $\mathrm{NH}_{3}$ ont été modélisées pour le cas des bâtiments d'élevage sur caillebottis. Ces émissions peuvent être réduites en diminuant la teneur en protéines de l'aliment distribué aux animaux ou en renouvelant fréquemment le lisier dans les bâtiments. Le traitement biologique du lisier permet aussi de réduire ces émissions, surtout en l'absence de séparation de phases. Le brassage du lisier dans les fosses de stockage les favorise au contraire. Les émissions de $\mathrm{NH}_{3}$ varient fortement en fonction du substrat utilisé pour la litière et de son mode de conduite. Pendant le compostage, elles dépendent aussi fortement des caractéristiques initiales du substrat composté, mais aussi de la technique utilisée.

Les émissions de $\mathrm{N}_{2} \mathrm{O}$ sont en général plus élevées avec de la litière que sur caillebotis, mais il y a de très fortes variations en fonction de son mode de conduite. Le compostage peut aussi conduire à des émissions de $\mathrm{N}_{2} \mathrm{O}$, tout particulièrement s'il est pratiqué en couche mince. Lors du traitement biologique des lisiers, ces émissions sont assez faibles mais dépendent de la technique d'aération utilisée. Dans le sol, les émissions de $\mathrm{N}_{2} \mathrm{O}$ ne sont pas plus importantes après apports d'effluents animaux qu'après apports de fertilisants minéraux et restent difficiles à quantifier.

Les émissions de $\mathrm{CH}_{4}$ sont plus faibles sur litière que sur caillebotis. Elles sont faibles également lors du compostage, sauf s'il est réalisé en couche mince. Le traitement biologique du lisier conduit à une forte réduction des émissions par rapport à un simple stockage.

Les litières et le compostage ont une excellente image de marque auprès du grand public et sont souvent réputées avoir beaucoup moins d'impact sur l'environnement que le lisier. Nous avons pu montrer cependant qu'elles génèrent en général davantage de gaz à effet de serre, que ce soit de façon directe (émissions de $\mathrm{N}_{2} \mathrm{O}$ ) ou indirecte (gaspillage de fertilisants azotés dont la synthèse est très «énergivore»), et ce d'autant plus que l'on cherche à rogner sur les coûts en augmentant la densité animale ou en diminuant la quantité de paille utilisée comme substrat de compostage. De même, le traitement biologique du lisier, qui semble à première vue avoir un impact favorable puisqu'il conduit à réduire les émissions de $\mathrm{NH}_{3}$ sans trop relarguer de $\mathrm{N}_{2} \mathrm{O}$, a en fait un bilan environnemental très défavorable du fait qu'il est très «énergivore» aussi bien directement qu'indirectement (gaspillage de fertilisants). On a là un double transfert de pollution, du sol et de l'eau vers l'atmosphère d'une part, et de l'exploitation vers l'extérieur de l'exploitation d'autre part.

\section{Abstract}

\section{Knowledge of Gas emissions in pig breeding}

This paper summarises the «Porcherie verte» programme in terms of knowledge and control of gas emissions occurring during the different phases of pig breeding. These emissions include green house gases, methane $\left(\mathrm{CH}_{4}\right)$ in particular and nitrous oxide $\left(\mathrm{N}_{2} \mathrm{O}\right)$, as well as ammoniac $\left(\mathrm{NH}_{3}\right)$, harmful for animals and man and having an impact on the environment in terms of acidification and eutrophication. $\mathrm{NH}_{3}$ emissions have been modelled for breeding buildings on wood slats. These emissions can be decreased by decreasing protein content of feed distributed to animals or by frequently renewing the manure in the buildings. The biological treatment of manure reduces these emissions, especially when there is no phase separation. The mixing of manure in storage tanks favours it. $\mathrm{NH}_{3}$ emissions vary strongly depending what substrate is used for litter and management. During composting, they also depend on the initial characteristics of the substrate composted, but also on the technique used.

$\mathrm{NO}_{2}$ emissions are generally higher with litter than with wood slate, but there are high variations depending on management. Composting can also lead to $\mathrm{NO}_{2}$ emissions, particularly with thin layers. When biological treatment of manure is used, these emissions are low but depend on the ventilation technique used. In the soil, $\mathrm{NO}_{2}$ emissions are not higher after adding animal waste than after adding mineral fertilisers and they are difficult to quantify.

$\mathrm{CH}_{4}$ emissions are lower on litter than on wood slats. They are also low with composting, except when thin layers are used. Biological treatment of manure leads to an important reduction in emissions as compared to storage. Litter and composting are often thought by the public to have a lower impact on the environment than manure. We have, however, shown that they generate more green house gases either directly (as $\mathrm{N}_{2} \mathrm{O}$ emissions) or indirectly (wasting of nitrogen fertilisers whose synthesis requires high amounts of energy). This is often accentuated since men try to lower costs by increasing animal density or decrease hay quantity used as a substrate for composting. Similarly, biological treatment of manure, which at first seems to have a favourable impact since it leads to lower $\mathrm{NH}_{3}$ emissions without releasing too much $\mathrm{N}_{2} \mathrm{O}$, actually has a poor environmental balance since it requires high amounts of energy either directly or indirectly (fertiliser waste). Indeed, there is twice as much pollution, in the soil and from water to the atmosphere on the one hand and from the farm to the environment on the other hand.

BONNEAU M., DOURMAD J.-Y., GERMON J.-C., HASSOUNA M., LEBRET B., LOYON L., PAILLAT J.-M., RAMONET Y., ROBIN P., 2008. Connaissance des émissions gazeuses dans les différentes filières de gestion des effluents porcins. INRA Prod. Anim., 21, 345-360. 
